\title{
Rational homological stability for groups of partially symmetric automorphisms of free groups
}

\author{
MATTHEW C B ZAREMSKY
}

Let $F_{n+m}$ be the free group of rank $n+m$, with generators $x_{1}, \ldots, x_{n+m}$. An automorphism $\phi$ of $F_{n+m}$ is called partially symmetric if for each $1 \leq i \leq m$, $\phi\left(x_{i}\right)$ is conjugate to $x_{j}$ or $x_{j}^{-1}$ for some $1 \leq j \leq m$. Let $\Sigma$ Aut $_{n}^{m}$ be the group of partially symmetric automorphisms. We prove that for any $m \geq 0$ the inclusion $\Sigma \mathrm{Aut}_{n}^{m} \rightarrow \Sigma \mathrm{Aut}_{n+1}^{m}$ induces an isomorphism in rational homology for dimensions $i$ satisfying $n \geq(3(i+1)+m) / 2$, with a similar statement for the groups $P \Sigma \mathrm{Aut}_{n}^{m}$ of pure partially symmetric automorphisms. We also prove that for any $n \geq 0$ the inclusion $\Sigma \mathrm{Aut}_{n}^{m} \rightarrow \Sigma \mathrm{Aut}_{n}^{m+1}$ induces an isomorphism in rational homology for dimensions $i$ satisfying $m>(3 i-1) / 2$.

20F65; 20F28, 57M07

\section{Introduction}

Let $\operatorname{Aut}\left(F_{n+m}\right)$ be the group of automorphisms of the free group $F_{n+m}$. For a fixed basis $\left\{x_{1}, \ldots, x_{n+m}\right\}$ of $F_{n+m}$, an automorphism $\phi$ of $F_{n+m}$ is called partially symmetric if for each $1 \leq i \leq m, \phi\left(x_{i}\right)$ is conjugate to $x_{j}$ or $x_{j}^{-1}$ for some $1 \leq j \leq m$. If $\phi$ is an automorphism such that each $\phi\left(x_{i}\right)$ is even conjugate to $x_{i}$ we call $\phi$ pure partially symmetric. Call these first $m$ generators distinguished and the other $n$ undistinguished. Let $\Sigma \mathrm{Aut}_{n}^{m}$ be the group of partially symmetric automorphisms of $F_{n+m}$, and $P \Sigma \mathrm{Aut}_{n}^{m}$ the group of pure partially symmetric automorphisms.

We prove that the rational homologies of $\Sigma \mathrm{Aut}_{n}^{m}$ and $P \Sigma \mathrm{Aut}_{n}^{m}$ are stable in the parameter $n$, and the rational homology of $\Sigma \mathrm{Aut}_{n}^{m}$ is also stable in $m$. This means that the rational homology is independent of the parameters once they are large enough. This question was posed by McEwen in his thesis [15], where a general strategy was outlined, involving a hypothetical Morse function on a version of a space introduced by Bux, Charney and Vogtmann [4]. As a first step, McEwen [15] and Zaremsky [16] construct a Morse function for the spine of Auter space, which provided a simplified proof of the so-called Degree theorem of Hatcher and Vogtmann [9]. From the Degree theorem, the rational homological stability of $\operatorname{Aut}\left(F_{n}\right)=\Sigma \mathrm{Aut}_{n}^{0}$ can be deduced. With this Morse-theoretic approach in hand for the classical case, it was supposed that one 
should then be able to generalize the situation to $\Sigma \mathrm{Aut}_{n}^{m}$, but this was left in the conjectural stage in [15]. In the present work we complete this project; namely, we exhibit a Morse function that yields a generalized version of the Degree theorem, from which we deduce rational homological stability for $\Sigma \mathrm{Aut}_{n}^{m}$.

To keep the notation straight, we mention that in [4] the "outer" version of the group we are calling $P \Sigma$ Aut $_{n}^{m}$ is denoted $P \Sigma(n, k)$, where $n$ is the total rank and $k$ the number of distinguished generators. Jensen and Wahl [14] denote the same group by $A_{n}^{k}$, where $n$ and $k$ are the number of undistinguished and distinguished generators, respectively. They also consider certain other groups denoted $A_{n, k}$, which are central extensions of $A_{n}^{k}$, but these are not the same as the groups $\Sigma \mathrm{Aut}_{n}^{m}$ considered here. For example, the automorphisms that properly permute the distinguished generators of $F_{n+m}$ appear only in $\Sigma \mathrm{Aut}_{n}^{m}$, and not in $P \Sigma \mathrm{Aut}_{n}^{m}=A_{n}^{m}$ or in $A_{n, m}$.

The relevant existing results are as follows. Hatcher and Vogtmann [9] showed that the homology of $\operatorname{Aut}\left(F_{n}\right)=\Sigma \mathrm{Aut}_{n}^{0}$ is stable with respect to $n$. In [7, Corollary 1.2], Galatius showed that the stable rational homology is even trivial, namely, $H_{i}\left(\operatorname{Aut}\left(F_{n}\right) ; \mathbb{Q}\right)=0$ for all $n>2 i+1$. At the other end of the spectrum, in [12] Hatcher and Wahl showed that the group of symmetric automorphisms $\Sigma \operatorname{Aut}\left(F_{m}\right)=\Sigma \operatorname{Aut}_{0}^{m}$ is homologically stable in $m$, and it turns out the rational homology actually vanishes in every dimension by independent results of Griffin [8] and Wilson [19]. In contrast, the pure case is quite different. The rational homology of $P \Sigma \mathrm{Aut}_{0}^{m}$ is not stable in $m$ [14], and in fact the cohomology ring has been completely computed by Jensen, McCammond and Meier [13]. To use the notation of [14], so $P \sum \operatorname{Aut}_{0}^{m}$ is denoted $A_{n}^{m}$, while the $A_{0}^{m}$ are not homologically stable, the groups $A_{n, m}$ are in fact stable in $n$ and $m$, even with coefficients in $\mathbb{Z}$, by work of Hatcher and Wahl [11]. We remark that the methods used to prove stability for $A_{n, m}$ are very different from how we will prove stability for $\Sigma \mathrm{Aut}_{n}^{m}$ here.

We actually obtain stability results for a range of families of subgroups of $\Sigma \mathrm{Aut}_{n}^{m}$, which includes the groups $P \sum$ Aut $_{n}^{m}$. Consider any family of groups $G_{n}^{m}$ such that

$$
P \Sigma \mathrm{Aut}_{n}^{m} \leq G_{n}^{m} \leq \Sigma \mathrm{Aut}_{n}^{m}
$$

for each $n$ and $m$, and such that the inclusion

$$
\Sigma \operatorname{Aut}_{n}^{m} \hookrightarrow \Sigma \mathrm{Aut}_{n+1}^{m},
$$

given by extending $\phi \in \Sigma$ Aut $_{n}^{m}$ to $F_{m+n+1}$ via $\phi\left(x_{n+m+1}\right)=x_{n+m+1}$, restricts to an inclusion $G_{n}^{m} \hookrightarrow G_{n+1}^{m}$. Of course $P \Sigma \mathrm{Aut}_{n}^{m}$ and $\Sigma \mathrm{Aut}_{n}^{m}$ are examples of such families of groups. Our main result for these groups is the following theorem. 
Theorem 1.1 (Stability in $n$ ) For any $m \geq 0$ and $i \geq 0$, and any family of groups $G_{n}^{m}$ satisfying the above conditions, the map

$$
H_{i}\left(G_{n}^{m} ; \mathbb{Q}\right) \rightarrow H_{i}\left(G_{n+1}^{m} ; \mathbb{Q}\right)
$$

induced by inclusion is an isomorphism for $n \geq(3(i+1)+m) / 2$.

Corollary The rational homology of $\Sigma \mathrm{Aut}_{n}^{m}$ is stable in $n$, as is the rational homology of P $\Sigma$ Aut $_{n}^{m}$.

We also consider stability in the other parameter, $m$. Renumber the elements of the basis by $\left\{x_{1}, \ldots, x_{n}, x_{n+1}, \ldots, x_{n+m}\right\}$, so an automorphism $\phi$ is partially symmetric if for all $1 \leq i \leq m, \phi\left(x_{n+i}\right)$ is conjugate to $x_{n+j}$ or $x_{n+j}^{-1}$ for some $1 \leq j \leq m$. We now have a natural inclusion map

$$
\Sigma \mathrm{Aut}_{n}^{m} \hookrightarrow \Sigma \mathrm{Aut}_{n}^{m+1},
$$

given by extending $\phi \in \Sigma$ Aut $_{n}^{m}$ to $F_{n+m+1}$ via $\phi\left(x_{n+m+1}\right)=x_{n+m+1}$.

Theorem 1.2 (Stability in $m$ ) For any $n \geq 0$ and $i \geq 0$, the map

$$
H_{i}\left(\Sigma \mathrm{Aut}_{n}^{m} ; \mathbb{Q}\right) \rightarrow H_{i}\left(\Sigma \mathrm{Aut}_{n}^{m+1} ; \mathbb{Q}\right)
$$

induced by inclusion is an isomorphism for $m>(3 i-1) / 2$.

In Section 2 we provide some background on the spine of Auter space $K_{n+m}$, and describe a contractible subcomplex $\Delta K_{n}^{m}$ that admits a nice $\Sigma \mathrm{Aut}_{n}^{m}$ action. We also filter $\Delta K_{n}^{m}$ using the notion of weighted degree, a generalization of degree from [9]. In Section 3 we define a height function $h$ on $\Delta K_{n}^{m}$, which generalizes the height function from the classical case, constructed by McEwen [15] and McEwen and Zaremsky [16]. We then show how the main result of Section 5, Proposition 5.14, about connectivity of descending links with respect to $h$, implies our so-called Generalized degree theorem, Theorem 3.5. In Section 4 we show how the Generalized degree theorem yields our homological stability results. In Section 5 we prove Proposition 5.14. This is done by separately considering two join factors, the $d$-down link, in Section 5.1, and the $d$-up link, in Section 5.2.

Acknowledgements The author is grateful to Kai-Uwe Bux for his guidance in this project, and to James Griffin, Rob McEwen and Jenny Wilson for their helpful advice along the way. This work was done while the author was supported by the SFB 701 in Bielefeld, Germany, and this support is gratefully acknowledged. Finally, many thanks are due to the anonymous referee, for a careful and thorough reading that resulted in a number of improvements to the paper. 


\section{Auter space and our space of interest}

We will analyze the homology of $\Sigma \mathrm{Aut}_{n}^{m}$ by considering its action on a certain simplicial complex. Our starting point is the well-studied spine of Auter space $K_{n}$ introduced by Hatcher and Vogtmann in [9]. Let $R_{n}$ be the rose with $n$ edges, ie the graph with a single vertex $p_{0}$ and $n$ edges. Here by a graph we mean a connected one-dimensional CWcomplex, with the usual notions of vertices and edges. We identify $F_{n}$ with $\pi_{1}\left(R_{n}\right)$. If $\Gamma$ is a graph with basepoint vertex $p$, a homotopy equivalence $\rho: R_{n} \rightarrow \Gamma$ is called a marking on $\Gamma$ if $\rho$ takes $p_{0}$ to $p$. We will consider two markings to be equivalent if there is a basepoint-preserving homotopy between them. We will be interested in the set of equivalence classes of triples $(\Gamma, p, \rho)$. We only consider graphs such that $p$ is at least bivalent and all other vertices are at least trivalent. Note that we do allow separating edges, that is edges whose complement in the graph is disconnected.

For graphs $\Gamma_{1}$ and $\Gamma_{2}$, a basepoint-preserving homotopy equivalence $d: \Gamma_{1} \rightarrow \Gamma_{2}$ is called a forest collapse or a blow-down if it amounts to collapsing a subforest $F$ of $\Gamma_{1}$. We write the blow-down as $\Gamma / F$. The reverse of a blow-down is, naturally, called a blow-up. This gives us a partial ordering on the set of equivalence classes of triples $(\Gamma, p, \rho)$, namely $\left(\Gamma^{\prime}, p, \rho^{\prime}\right) \leq(\Gamma, p, \rho)$ if there is a forest collapse $d: \Gamma \rightarrow \Gamma^{\prime}$ such that $\rho^{\prime}$ is equivalent to $d \circ \rho$. The spine $K_{n}$ of Auter space is then the geometric realization of the poset of equivalence classes of triples $(\Gamma, p, \rho)$ with $\Gamma$ a rank- $n$ graph, with this partial ordering. In particular the vertices of $K_{n}$ are equivalence classes of marked basepointed graphs.

Since we are identifying $F_{n}$ with $\pi_{1}\left(R_{n}\right)$, we can also identify $\operatorname{Aut}\left(F_{n}\right)$ with the group of basepoint-preserving homotopy equivalences of $R_{n}$, up to homotopy. This is the same as the group of markings of $R_{n}$, so we can denote markings on $R_{n}$ by elements of $\operatorname{Aut}\left(F_{n}\right)$. There is a (right) action of $\operatorname{Aut}\left(F_{n}\right)$ on $K_{n}$ in the following way: given a vertex $(\Gamma, p, \rho)$ in $K_{n}$ and $\phi \in \operatorname{Aut}\left(F_{n}\right)$, we have

$$
\phi(\Gamma, p, \rho)=(\Gamma, p, \rho \circ \phi) .
$$

This action only affects markings, and in fact $\operatorname{Aut}\left(F_{n}\right)$ permutes markings arbitrarily.

\section{A space for $\Sigma \mathrm{Aut}_{\boldsymbol{n}}^{\boldsymbol{m}}$}

We now describe a subcomplex of $K_{n+m}$ on which $\Sigma \mathrm{Aut}_{n}^{m}$ acts nicely. First we will restrict to only allowing certain markings, using a standard technique, and then we will restrict further to only allowing certain graphs, à la Bux, Charney and Vogtmann [4].

Let $\left(R_{n+m}, p_{0}, \phi\right)$ be a marked rose in $K_{n+m}$, so the marking $\phi$ is really an element of $\operatorname{Aut}\left(F_{n+m}\right)$. Let $W$ be the set of conjugacy classes in $F_{n+m}$ of the distinguished 
generators and their inverses, $x_{1}^{ \pm 1}, \ldots, x_{m}^{ \pm 1}$. Note that $\operatorname{Aut}\left(F_{n+m}\right)$ acts on the set of all conjugacy classes, and $\Sigma$ Aut $_{n}^{m}$ is precisely the stabilizer of $W$. We say that the rose $\left(R_{n+m}, p_{0}, \phi\right)$ has minimal norm if the quantity

$$
\sum_{w \in W}|\phi(w)|
$$

is minimized, where $|\phi(w)|$ is the length of a cyclically reduced representative of the conjugacy class $\phi(w)$, with respect to the generating set $\left\{x_{i}^{ \pm 1}\right\}_{i=1}^{n+m}$. The star of this rose is the subcomplex of $K_{n+m}$ spanned by vertices that are obtained by blowing up the rose. Denote by $K_{n+m}^{W}$ the subcomplex of $K_{n+m}$ that is the union of the stars of all roses with minimal norm.

Similar complexes, for other $W$, were crucial to the original proof of contractibility of Outer space by Culler and Vogtmann [6]. Our $K_{n+m}^{W}$ was considered by McEwen in [15], and the "Outer" version was considered in [4, Section 3.1]. The complex $K_{n+m}^{W}$ is contractible and $\Sigma \mathrm{Aut}_{n}^{m}$-invariant. If the marked basepointed graph $(\Gamma, p, \rho)$ lies in $K_{n+m}^{W}$ we will call the marking $\rho$ admissible.

Having restricted to admissible markings, we next impose restrictions on the graphs. Let $(\Gamma, p, \rho)$ be a vertex in $K_{n+m}^{W}$, so $\rho: R_{n+m} \rightarrow \Gamma$ is an admissible marking. We consider the closed paths, or cycles, $\rho\left(x_{i}\right)$ in $\Gamma$ for $1 \leq i \leq m$. These cycles may not be reduced, so for each $1 \leq i \leq m$ let $C_{i}$ be the reduced cycle in $\Gamma$ obtained by reducing $\rho\left(x_{i}\right)$. Here reducing a path means inductively removing any subpaths consisting of an edge followed immediately by the same edge in the reverse direction, and a path is reduced if no such reduction is possible. Since $\rho$ is admissible, $C_{i}$ is an embedded simple cycle, that is, it is homeomorphic to a circle; this is for the same reason as in the nonbasepointed case [4, Lemma 15(1)].

Definition 2.1 (Viable graph) With the above notation, we call a graph $\Gamma$ viable if the $C_{i}$ are all pairwise disjoint.

See Figure 1 for an example. For brevity we will just define a viable marked graph to be a viable graph with an admissible marking. Let $\Delta K_{n}^{m}$ be the subcomplex of $K_{n+m}^{W}$ consisting of viable marked graphs. Compare this to the nonbasepointed version, denoted $D_{n, k}$, in [4].

The cycles $C_{i}$ for $1 \leq i \leq m$ are called distinguished cycles, and we similarly refer to vertices, edges, half-edges and edge paths as distinguished if they are contained in some $C_{i}$. A forest $F$ in a viable marked graph $\Gamma$ is called admissible if $\Gamma / F$ is again viable and the induced marking is again admissible, ie, it is still a point in $\Delta K_{n}^{m}$. 
Lemma 2.2 (Admissible trees) Let $F$ be an admissible forest in $\Gamma$ and $T$ a tree in $F$. Then $T$ intersects at most one distinguished cycle $C$, and if $T \cap C$ is nonempty then it must either be a single vertex or a connected edge path in $C$. Moreover, if $F$ is a forest such that every tree in $F$ satisfies this property, then $F$ is admissible.

Proof If $T$ meets two distinguished cycles, $C$ and $C^{\prime}$, then the images of these cycles in $\Gamma / F$ meet, violating viability. Now suppose $T \cap C \neq \varnothing$. The image of $C$ in $\Gamma / F$ must be homeomorphic to $S^{1}$; this tells us that $T \cap C$ is connected. But then the only options are a single vertex or a connected edge path.

For the converse, we need only observe that blowing down a tree that meets a single distinguished cycle in a connected subspace yields a viable graph.

An example of an admissible and an inadmissible forest (for some marking $\rho$ ) are shown in gray in Figure 1.
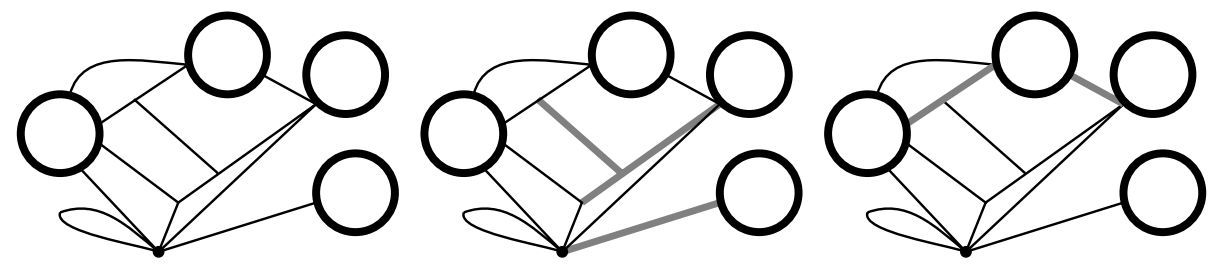

Figure 1: From left to right: a viable graph, an admissible forest and an inadmissible forest

The action of $\Sigma \mathrm{Aut}_{n}^{m}$ on $K_{n+m}^{W}$ only affects markings, so we can consider the action of $\Sigma \mathrm{Aut}_{n}^{m}$ on $\Delta K_{n}^{m}$. Let

$$
\Delta Q_{n}^{m}:=\Delta K_{n}^{m} / \Sigma \mathrm{Aut}_{n}^{m}
$$

be the orbit space.

Proposition 2.3 The subcomplex $\Delta K_{n}^{m}$ is contractible, and $\Sigma \mathrm{Aut}_{n}^{m}$ acts on $\Delta K_{n}^{m}$ with finite stabilizers and finite quotient $\Delta Q_{n}^{m}$.

Proof There is an equivariant deformation retraction of $K_{n+m}^{W}$ onto $\Delta K_{n}^{m}$. This follows by a parallel argument to the proofs of Propositions 16 and 17 in [4]. The only difference is that our graphs have basepoints, but all the arguments carry through. That the stabilizers are finite follows because the stabilizers in $\operatorname{Aut}\left(F_{n+m}\right)$ of vertices in $K_{n+m}$ are already finite. Lastly, $\Sigma \mathrm{Aut}_{n}^{m}$ is transitive on markings of a given (viable) graph, and there are only finitely many homeomorphism types of graphs with rank $n+m$, so $\Delta Q_{n}^{m}$ is finite. 
If an element of $\Sigma \mathrm{Aut}_{n}^{m}$ stabilizes a simplex then it fixes it pointwise, since the vertices of any simplex correspond to pairwise nonisomorphic graphs. This, together with the previous proposition, imply that $\Delta Q_{n}^{m}$ and $\Sigma \mathrm{Aut}_{n}^{m}$ have the same rational homology; see for example Brown [2, Exercise 2, page 174].

\section{A useful further reduction}

There is a nice subcomplex of $\Delta K_{n}^{m}$ that will prove useful for our purposes, namely the subcomplex $\nabla K_{n}^{m}$ spanned by marked basepointed graphs in $\Delta K_{n}^{m}$ in which the basepoint $p$ is not contained in a distinguished cycle. The action of $\Sigma \mathrm{Aut}_{n}^{m}$ on $\nabla K_{n}^{m}$ similarly features finite stabilizers and finite quotient

$$
\nabla Q_{n}^{m}:=\nabla K_{n}^{m} / \Sigma \operatorname{Aut}_{n}^{m} .
$$

To keep straight which is which notationally, note that the symbol $\nabla$ is "top heavy" compared to $\Delta$, indicating that the distinguished cycles cannot be down at the basepoint.

\section{Weighted degree}

It is difficult to analyze $\Delta Q_{n}^{m}$ and $\nabla Q_{n}^{m}$ directly, and so we will work with a certain filtration. For a vertex $(\Gamma, p, \rho)$ in $\Delta K_{n}^{m}$, define the weighted valency $\operatorname{val}_{w}(v)$ of a vertex $v$ to be the number of undistinguished half-edges at $v$, plus half the number of distinguished half-edges. Define the weighted degree $d_{w}(\Gamma)$ to be

$$
d_{w}(\Gamma):=2 n+m-\operatorname{val}_{w}(p) .
$$

Note that $1 \leq \operatorname{val}_{w}(p) \leq 2 n+m$, and so $0 \leq d_{w}(\Gamma) \leq N$, where $N:=2 n+m-1$. As an example, the reader can verify that the weighted degree of the graph in Figure 1 is 10. We will also make use of the notion of degree from Hatcher and Vogtmann [9], which we define to be

$$
d_{0}(\Gamma):=2 n+2 m-\operatorname{val}(p) .
$$

If $c$ denotes the number of distinguished cycles not containing $p$ then $d_{w}=d_{0}-c$. The reader curious about the motivation for defining weighted degree this way should glance ahead to the paragraph after Definition 4.2.

For $k \in \mathbb{N}_{0}$ let $\Delta K_{n, k}^{m}$ be the full subcomplex of $\Delta K_{n}^{m}$ spanned by marked basepointed graphs with weighted degree less than or equal to $k$. In particular for $k \geq N$, $\Delta K_{n, k}^{m}=\Delta K_{n}^{m}$. Also let $\nabla K_{n, k}^{m}=\Delta K_{n, k}^{m} \cap \nabla K_{n}^{m}$. The sequence of spaces

$$
\nabla K_{n, 0}^{m} \subseteq \nabla K_{n, 1}^{m} \subseteq \cdots
$$


is a filtration of $\nabla K_{n}^{m}$, and not of the whole contractible complex $\Delta K_{n}^{m}$, but these smaller complexes will prove to be the right ones to inspect for various reasons. Note that when $m=0, \nabla K_{n, k}^{0}=\Delta K_{n, k}^{0}=K_{n, k}$, the filtration of $K_{n}$ by degree used in [9]. As a bit of foreshadowing to Section 4, note that the undistinguished loop and/or the distinguished loop on a stick (or "lollipop") at the basepoint in Figure 1 could be removed without changing the weighted degree. This property is precisely the motivation for filtering $\Delta K_{n}^{m}$ and $\nabla K_{n}^{m}$ by weighted degree.

At this point we can state the Generalized degree theorem, which we will prove in the next section.

Theorem 3.5 (Generalized degree theorem) For each $0 \leq k<N, \nabla K_{n, k}^{m}$ is $(k-1)$ connected.

A lot of notation has been introduced by now, some of which will not appear again for a while, so for reference we collect it here. All the notation in the table will be considered fixed for the rest of the paper.

\begin{tabular}{|c|c|}
\hline Symbol & Meaning \\
\hline$m$ & number of distinguished generators \\
\hline$n$ & number of undistinguished generators \\
\hline$p$ & basepoint \\
\hline$c$ & number of distinguished cycles not at $p$ \\
\hline$K_{n+m}$ & space of all marked graphs \\
\hline$\Delta K_{n}^{m}$ & only allow admissible markings and viable graphs \\
\hline$\nabla K_{n}^{m}$ & same as above, plus no distinguished cycles at basepoint \\
\hline$C_{1}, \ldots, C_{m}$ & distinguished cycles \\
\hline $\operatorname{val}(v)\left(\operatorname{resp} . \operatorname{val}_{w}(v)\right)$ & valency (resp. weighted valency) of vertex $v$ \\
\hline$d_{0}(\Gamma)\left(\operatorname{resp} . d_{w}(\Gamma)\right)$ & degree (resp. weighted degree) of graph $\Gamma$ \\
\hline $\begin{array}{l}\nabla K_{n, k}^{m} \\
\nabla O^{m}\end{array}$ & $\begin{array}{l}\text { points of } \nabla K_{n}^{m} \text { with weighted degree at most } k \\
\text { the quotient of } \nabla K^{m} \text { by } \Sigma \text { Aut }{ }_{n}^{m}\end{array}$ \\
\hline$z_{n, k}$ & 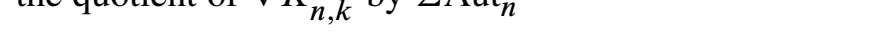 \\
\hline
\end{tabular}

Table 1: Notation

\section{A height function}

We now define a height function $h$ on the vertices of $\Delta K_{n}^{m}$. This height function is related to the one defined by McEwen and Zaremsky in [16] on the space $K_{n}=\Delta K_{n}^{0}$. This will allow us to inspect the connectivity of $\nabla K_{n, k}^{m}$ using discrete Morse theory; see Bestvina and Brady [1] for background on discrete Morse theory. 
Definition 3.1 (Features of graphs) Let $(\Gamma, p, \rho)$ be a basepointed viable marked graph. For vertices $v, v^{\prime}$ in $\Gamma$, let the distance $d\left(v, v^{\prime}\right)$ be the number of edges in a minimal length edge path from $v$ to $v^{\prime}$. Also, for a subforest $F$ of $\Gamma$, define the level $D(F)$ of $F$ to be the smallest $i$ such that $F$ has a vertex at distance $i$ from $p$. Let

$$
\Lambda_{i}(\Gamma):=\{v \in \Gamma \mid d(p, v)=i\}
$$

be the $i^{\text {th }}$ level of $\Gamma$, so for example $\Lambda_{0}(\Gamma)=\{p\}$. If $v$ is a vertex that is in a distinguished cycle $C$, and $d(p, v) \leq d\left(p, v^{\prime}\right)$ for any other vertex $v^{\prime}$ in $C$, then we will say that $v$ is a base vertex for $C$, and call $i_{C}:=d(p, v)$ the base height of $C$. If $v$ is a base vertex for some $C$, call $v$ a base vertex.

Note that the basepoint $p$ is a base vertex if and only if it is distinguished, if and only if $c=m-1$, where recall that $c$ is defined as the number of distinguished cycles not containing $p$. In Figure 2 the distinguished cycle $C$ is indicated by thick edges, the base vertices are the larger dots, and the basepoint is the largest dot at the bottom.

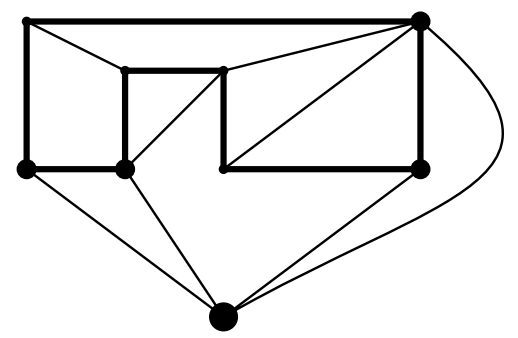

Figure 2: Distinguished cycle $C$ with $i_{C}=1$

\section{Measurements contributing to the height function}

For each $i \geq 0$, let $m_{i}(\Gamma)$ denote the number of base vertices in $\Lambda_{i}(\Gamma)$, define $n_{i}(\Gamma):=-\left|\Lambda_{i}(\Gamma)\right|$ and let

$$
d_{i}(\Gamma):=\sum_{v \notin \Lambda_{i}}(\operatorname{val}(v)-2) .
$$

Note that $m_{0}=m-c, n_{0}$ is constant -1 and $d_{0}=2 n+2 m-\operatorname{val}(p)$ is the degree. In general $d_{i}$ can be thought of as counting the number of vertices not at level $i$, with higher valence vertices "counting for more." Now define

$$
h_{i}(\Gamma):=\left(m_{i}(\Gamma), n_{i}(\Gamma), d_{i}(\Gamma)\right) \quad \text { and set } \quad h(\Gamma)=\left(h_{0}(\Gamma), h_{1}(\Gamma), h_{2}(\Gamma), \ldots\right)
$$

with the lexicographic order. We remark that the height function used in [16] on the spine of Auter space was $\left(d_{0}, n_{1}, d_{1}, n_{2}, d_{2}, \ldots\right)$, which encodes the same information 
as our $h$ when $m=0$. Extend $h$ to the vertices of $\Delta K_{n}^{m}$ via $h(\Gamma, p, \rho)=h(\Gamma)$. In general we will just write $\Gamma$ to denote vertices of $\Delta K_{n}^{m}$, with the basepoint and marking understood.

\section{How forests affect the measurements}

We need to understand how our height function changes upon blowing down a forest. The first thing to check is that it does indeed change.

Lemma 3.2 (Height always changes) Let $F$ be an admissible forest in $\Gamma$. Then blowing down $F$ either increases or decreases $h_{D(F)}$. More precisely, blowing down $F$ either increases $n_{D(F)}$, or else does not change $n_{D(F)}$ and decreases $d_{D(F)}$. Also, blowing down $F$ does not change any $h_{i}$ for $i<D(F)$.

Proof First consider how the $d_{i}$ are affected by blowing down a single edge $\varepsilon$. The endpoints of the edge become a single vertex, whose valency equals the sum of the valencies of the endpoints, minus two. If neither endpoint of $\varepsilon$ lies in $\Lambda_{i}$, then the $\operatorname{sum} \sum_{w \notin \Lambda_{i}} \operatorname{val}(w)$ drops by 2 upon blowing down $\varepsilon$. It also decreases the number of vertices not in $\Lambda_{i}$ by one, which implies that the sum $d_{i}=\sum_{w \notin \Lambda_{i}}(\operatorname{val}(w)-2)$ does not change. If one endpoint of $\varepsilon$ lies in $\Lambda_{i}$ and the other in $\Lambda_{i+1}$, then blowing down $\varepsilon$ just eliminates one term of $d_{i}$, strictly decreasing it. Finally, if both endpoints of $\varepsilon$ lie in $\Lambda_{i}$ then blowing down $\varepsilon$ does not change $d_{i}$.

Now consider the forest $F$. If $F$ connects vertices in $\Lambda_{D(F)}$ then blowing down $F$ decreases the number of such vertices and hence increases $n_{D(F)}$. Now suppose $F$ does not connect any vertices in $\Lambda_{D(F)}$, so blowing down $F$ does not change $n_{D(F)}$. Then $F$ must connect a vertex that is not in $\Lambda_{D(F)}$ to a vertex that is in $\Lambda_{D(F)}$. Upon blowing down $F$ one edge at a time (in any order), we will eventually blow down an edge connecting a vertex at level $D(F)$ to one at level $D(F)+1$, and so we conclude that blowing down $F$ strictly decreases $d_{D(F)}$.

Now let $i<D(F)$. Blowing down $F$ induces a bijection $f: \Lambda_{i}(\Gamma) \rightarrow \Lambda_{i}(\Gamma / F)$, and moreover $f(v)$ is a base vertex if and only if $v$ is. This tells us that $m_{i}$ and $n_{i}$ are unchanged upon blowing down $F$. That $d_{i}$ is unchanged follows from the first paragraph.

In general, of all the terms in $h$ changed by blowing down $F$, there is one that is lexicographically first, which we will call the essential term of $F$. Similarly, any blow-up has an essential term.

It will be important in the future to know some restrictions on the ways in which the various measurements can possibly change. 
Observation 3.3 (Some restrictions) A blow-down at level $i$ cannot decrease $n_{i}$, and a blow-up at level $i$ cannot decrease $d_{i}$, though both blow-downs and blow-ups can either increase or decrease $m_{i}$.

Proof The first and last statements need no explanation (though perhaps we should reiterate that $n_{i}$ is the negative of $\left|\Lambda_{i}\right|$ ). The second statement follows from the proof of Lemma 3.2, which says that a blow-down at level $i$ cannot increase $d_{i}$.

Lemma 3.4 (Sublevel sets) The complex $\nabla K_{n}^{m}$ is the sublevel set of $\Delta K_{n}^{m}$ defined by the inequality

$$
h \leq(0,0,0, \ldots) \text {. }
$$

Also, $\nabla K_{n, k}^{m}$ is the sublevel set of $\Delta K_{n}^{m}$ defined by

$$
h \leq(0,-1, k+m+1,-1,0, \ldots) .
$$

Proof If $h(\Gamma) \leq(0,0,0, \ldots)$ then in particular $m_{0}(\Gamma)=0$ and so $\Gamma \in \nabla K_{n}^{m}$. Conversely, if $\Gamma \in \nabla K_{n}^{m}$ then $m_{0}(\Gamma)=0$, and so $h(\Gamma)=\left(0,-1, d_{0}(\Gamma), \ldots\right) \leq(0,0,0, \ldots)$. This proves the first claim.

Now suppose $h(\Gamma) \leq(0,-1, k+m+1,-1,0, \ldots)$, so $m_{0}(\Gamma)=0$ and $d_{0}(\Gamma) \leq k+m+1$. Since $m_{0}=0$ we have $d_{0}=d_{w}+m$, so $d_{w}(\Gamma) \leq k+1$. If this is even an equality, that is if $d_{w}(\Gamma)=k+1$, then $h(\Gamma)=\left(0,-1, k+m+1, m_{1}(\Gamma), \ldots\right)$ and so $m_{1}(\Gamma) \leq-1$, which is absurd. Thus in fact $d_{w}(\Gamma) \leq k$ and so $\Gamma \in \nabla K_{n, k}^{m}$. Finally suppose $\Gamma \in \nabla K_{n, k}^{m}$. Then $m_{0}(\Gamma)=0$ and $d_{w}(\Gamma) \leq k$. Hence $d_{0}(\Gamma) \leq k+m$, and so we conclude that $h(\Gamma) \leq(0,-1, k+m+1,-1,0, \ldots)$.

The upshot of Lemmas 3.2 and 3.4 is that connectivity of $\nabla K_{n, k}^{m}$ can be determined by looking at descending links of vertices with respect to $h$. For a vertex $\Gamma$ in $\Delta K_{n}^{m}$, the descending star st $\downarrow(\Gamma)$ with respect to $h$ is the set of simplices in the star of $\Gamma$ whose other vertices all have strictly lower height than $\Gamma$. The descending link $1 \mathrm{k} \downarrow(\Gamma)$ consists of the faces of simplices in st $\downarrow(\Gamma)$ that do not themselves contain $\Gamma$.

The proof of the following main result will make up all of Section 5.

Proposition 5.14 (Connectivity of descending links) The descending link $1 \mathrm{k} \downarrow(\Gamma)$ is either contractible or a wedge of spheres of dimension $d_{w}(\Gamma)-1$.

From this, our so-called Generalized degree theorem stated below follows quickly, as we now show. Recall that the weighted degree $d_{w}$ of a graph can never exceed $N=2 n+m-1$. Moreover, $d_{w}=N$ if and only if the basepoint $p$ has valency 2 and is a base vertex. In addition to Proposition 5.14, we will need to use Lemma 5.12, which will also be proved later, but we state a relevant version of it here for reference. 
Lemma 5.12 (Contractible case) If $p$ is a base vertex and $\operatorname{val}(v)>2$, then $1 \mathrm{k} \downarrow(\Gamma)$ is contractible.

Theorem 3.5 (Generalized degree theorem) For each $0 \leq k<N, \nabla K_{n, k}^{m}$ is $(k-1)-$ connected.

Proof Since $\Delta K_{n}^{m}$ is contractible, it suffices by [1, Corollary 2.6] to show that for any vertex $\Gamma$ in $\Delta K_{n}^{m} \backslash \nabla K_{n, k}^{m}$, the descending link $1 \mathrm{k} \downarrow(\Gamma)$ is at least $(k-1)$-connected. Let $\Gamma$ be such a vertex, so either $d_{w}(\Gamma)>k$, or else $d_{w}(\Gamma) \leq k$ and $m_{0}(\Gamma)=1$. In the former case, $1 \mathrm{k} \downarrow(\Gamma)$ is $\left(d_{w}-2\right)$-connected by Proposition 5.14 , and hence $(k-1)$-connected. In the latter case, the basepoint $p$ is a base vertex, and since $d_{w}(\Gamma)<N, \operatorname{val}(p)>2$, so by Lemma $5.12,1 \mathrm{k} \downarrow(\Gamma)$ is contractible.

In the rest of this section we do some preliminary work with $\operatorname{lk} \downarrow(\Gamma)$. Then in Section 4 we show how the Generalized degree theorem gives us homological stability results. Finally in Section 5 we prove Proposition 5.14, and, along the way, Lemma 5.12.

\section{The d-up link and d-down link}

There are two types of vertices in $\operatorname{lk} \downarrow(\Gamma)$ : those obtained from $\Gamma$ by a descending blow-up, and those obtained by a descending blow-down. Here we say that a blow-up or blow-down is descending if the resulting graph has a lower height than the starting graph. Call the subcomplex of $\operatorname{lk} \downarrow(\Gamma)$ spanned by vertices of the first type the $d$-up link, and the subcomplex spanned by vertices of the second type the $d$-down link. Any vertex in the d-up link is related to every vertex in the d-down link by a blow-down, so $1 \mathrm{k} \downarrow(\Gamma)$ is the simplicial join of the d-up- and d-down links. We remark that we only consider admissible blow-downs, and on the other hand observe that any blow-up of a viable graph is again viable. If a forest blow-down is descending we call the forest itself descending, and similarly we refer to ascending forests. By Lemma 3.2, every forest is either descending or ascending.

It will be important to have a somewhat explicit description of which forests are descending.

Lemma 3.6 (Interpreting the height function $h$ ) Let $F$ be an admissible forest in $\Gamma$ with $i:=D(F)$.

(i) If $m_{i}(\Gamma / F)<m_{i}(\Gamma)$, then $F$ is descending.

(ii) If $m_{i}(\Gamma / F)>m_{i}(\Gamma)$, then $F$ is ascending.

(iii) If $m_{i}(\Gamma / F)=m_{i}(\Gamma)$ and $F$ connects vertices in $\Lambda_{i}$, then $F$ is ascending.

(iv) If $m_{i}(\Gamma / F)=m_{i}(\Gamma)$ and $F$ does not connect vertices in $\Lambda_{i}$, then $F$ is descending. 
Proof The essential term of $F$ occurs in $h_{i}$ (Lemma 3.2), so the first two claims are immediate. Suppose $m_{i}(\Gamma / F)=m_{i}(\Gamma)$. If $F$ connects vertices in $\Lambda_{i}$, then blowing down $F$ increases $n_{i}$ (Lemma 3.2) and so is ascending. If $F$ does not connect vertices in $\Lambda_{i}$, then blowing down $F$ does not change $n_{i}$, but decreases $d_{i}$ (Lemma 3.2), so $F$ is descending.

Again we have accumulated a lot of notation, which we collect in a table for easy reference.

\begin{tabular}{c|l} 
Symbol & Meaning \\
\hline$D(F)$ & level of $F$ \\
$\Lambda_{i}(\Gamma)$ & $i^{\text {th }}$ level of $\Gamma$ \\
$i_{C}$ & base height of distinguished cycle $C$, ie level of any base vertex of $C$ \\
$m_{i}$ & number of base vertices in $\Lambda_{i}$ \\
$n_{i}$ & negative number of vertices in $\Lambda_{i}$ \\
$d_{i}$ & sum of valencies minus two of vertices not in $\Lambda_{i}$ \\
$h_{i}$ & $i^{\text {th }}$ term of height function $h$ \\
$\operatorname{lk} \downarrow(\Gamma)$ & descending link of $\Gamma$ in $\Delta K_{n}^{m}$ with respect to $h$
\end{tabular}

Table 2: More notation

\section{Homological stability}

As in Section 1, let $G_{n}^{m}$ be any family of groups such that $P \Sigma \mathrm{Aut}_{n}^{m} \leq G_{n}^{m} \leq \Sigma \mathrm{Aut}_{n}^{m}$ for each $n$ and $m$, and such that the inclusion $\Sigma \mathrm{Aut}_{n}^{m} \hookrightarrow \Sigma \mathrm{Aut}_{n+1}^{m}$ restricts to an inclusion $G_{n}^{m} \hookrightarrow G_{n+1}^{m}$. For any $0 \leq k<N$, the action of $G_{n}^{m}$ on $\nabla K_{n, k}^{m}$ has finite stabilizers and finite quotient $\nabla K_{n, k}^{m} / G_{n}^{m}$. Hence by the Generalized degree theorem, $\nabla K_{n, k}^{m} / G_{n}^{m}$ has the same rational homology as $G_{n}^{m}$ in dimensions $i$ with $i<k$. To be precise, we have the following.

Lemma 4.1 (From groups to orbit spaces) For any $0 \leq k<N$, we have that $H_{i}\left(\nabla K_{n, k}^{m} / G_{n}^{m} ; \mathbb{Q}\right)$ is isomorphic to $H_{i}\left(G_{n}^{m} ; \mathbb{Q}\right)$ for $i<k$, and $H_{k}\left(\nabla K_{n, k}^{m} / G_{n}^{m} ; \mathbb{Q}\right)$ surjects onto $H_{k}\left(G_{n}^{m} ; \mathbb{Q}\right)$.

To get homological stability in $n$ for $G_{n}^{m}$ we can now look for homological stability in $n$ for $\nabla K_{n, k}^{m} / G_{n}^{m}$. We will do this in a similar way as was done in the classical $m=0$ case by Hatcher and Vogtmann [9, Section 5]. The vertices of $\nabla K_{n}^{m} / P \Sigma$ Aut $_{n}^{m}$ are the homeomorphism types of basepointed graphs with $m$ distinguished oriented cycles, disjoint and distinguishable from each other and disjoint from the basepoint. In 
$\nabla K_{n, k}^{m} / \Sigma \mathrm{Aut}_{n}^{m}$ the cycles become nonoriented and indistinguishable from each other, and in general $\nabla K_{n, k}^{m} / G_{n}^{m}$ interpolates between these two extremes. Exactly as in [9], we have a map

$$
\text { v: } \nabla K_{n, k}^{m} / G_{n}^{m} \hookrightarrow \nabla K_{n+1, k}^{m} / G_{n+1}^{m}
$$

induced by sending a graph $\Gamma$ to $\Gamma \vee S^{1}$, that is the graph with an extra (undistinguished) loop wedged to its basepoint.

To get stability in $n$, we want to be able to "detect" loops and theta subgraphs at the basepoint. If $\Gamma$ has a loop at the basepoint $p$ then $\Gamma$ is in the image of $v$, which is why want to be able to detect loops. We will see in Proposition 4.5 why theta subgraphs at the basepoint are also useful.

First we set up the situation for stability in $m$. Instead of loops and theta subgraphs we will use certain subgraphs defined as follows.

Definition 4.2 (Lollipops and double lollipops) A lollipop in $\Gamma$ is a subgraph $\ell$ consisting of an undistinguished nonloop edge $\varepsilon$ (the stick) and a distinguished loop $\delta$ sharing a vertex $v \neq p$, such that $\varepsilon$ and $\delta$ are the only edges incident to $v$.

To define double lollipops we first make precise the notion of an $r$-iterated lollipop wedge. Take a collection of lollipops $\ell_{1}, \ldots, \ell_{r}$, with their univalent vertices specified as basepoints. Set $W_{1}=\ell_{1}$. Assuming $W_{i}$ has been constructed, we let $W_{i+1}$ be the result of wedging $\ell_{i+1}$, at its basepoint, onto $W_{i}$ at any point of $W_{i}$, even perhaps a nonvertex point. A double lollipop then is a subgraph of $\Gamma$ that is a 2 -iterated lollipop wedge.

Define a map

$$
\mu: \nabla K_{n, k}^{m} / \Sigma \operatorname{Aut}_{n}^{m} \hookrightarrow \nabla K_{n, k}^{m+1} / \Sigma \operatorname{Aut}_{n}^{m+1}
$$

by sending $\Gamma$ to $\Gamma \vee \ell$, where $\ell$ is a lollipop wedged to the basepoint. Unlike attaching an undistinguished loop, attaching a lollipop in this way changes the degree, but it does not change the weighted degree, so this is still fine. (Indeed this was precisely the impetus for defining weighted degree as we did.) We now describe how to detect the presence of these various subgraphs at the basepoint, as in [9, Lemma 5.2]. Following that, we will see why this gives us stability.

Lemma 4.3 (Detecting features at the basepoint) Let $\Gamma$ be a graph with basepoint $p$, rank $n+m$, weighted degree $d_{w}$, and $m$ pairwise disjoint distinguished cycles, disjoint from $p$. The following hold: 
(i) If $n>2 d_{w}+m$ then $\Gamma$ has a loop at the basepoint.

(ii) If $n>\left(3 d_{w}+m\right) / 2$ then $\Gamma$ has either a loop at the basepoint or a theta graph wedge summand.

(iii) If $m>2 d_{w}$ then $\Gamma$ has a lollipop at the basepoint.

(iv) If $m>3 d_{w} / 2$ then $\Gamma$ has a lollipop or a double lollipop at the basepoint.

Proof Since $p$ is not contained in a distinguished cycle, we have that the degree $d_{0}$ is $d_{0}=d_{w}+m$. The first two parts of the lemma then follow from [9, Lemma 5.2]. Next suppose that there are no lollipops at $p$, and we want to show that $m \leq 2 d_{w}+1$. We will induct on $n$. If $n=0$ then every undistinguished edge in $\Gamma$ is a separating edge. Let $\Gamma^{\prime}$ be the graph obtained by blowing down every undistinguished edge. Now $\Gamma^{\prime}$ is a cactus graph as in Collins [5], ie every edge is contained in a unique reduced cycle. Note that $\Gamma^{\prime}$ is no longer in $\Delta K_{0}^{m}$, since the distinguished cycles are not disjoint, but $\Gamma^{\prime}$ has the same weighted degree $d_{w}$ as $\Gamma$. Let $b^{\prime}$ be the number of cycles in $\Gamma^{\prime}$ at $p$ and $c^{\prime}=m-b^{\prime}$ the number of cycles not at $p$. Since $\Gamma$ had no lollipops (or loops) at $p, \Gamma^{\prime}$ has no loops at $p$. This tells us that $b^{\prime} \leq c^{\prime}$, and since $m=b^{\prime}+c^{\prime}$ we see that $m \leq 2 c^{\prime}$. Also, in $\Gamma^{\prime}, c^{\prime}=m-\operatorname{val}(p) / 2=d_{w}$, so indeed $m \leq 2 d_{w}$. This finishes the base case, and we also note that if additionally $\Gamma$ has no double lollipops then $b^{\prime} \leq c^{\prime} / 2$, so $m \leq 3 c^{\prime} / 2=3 d_{w} / 2$.

Now assume $n>0$. Then there exists an undistinguished edge $\varepsilon$ that is not a separating edge. Let $\Gamma_{1}$ be the graph obtained from $\Gamma$ by removing $\varepsilon$, and then if any bivalent vertices $v \neq p$ arise (or univalent vertices $v$ ), blowing down one of the edges containing $v$. Then $\Gamma_{1}$ is a connected graph with undistinguished rank $n-1$ and $m$ distinguished cycles. Let $a \in\{0,1,2\}$ be such that the weighted degree $d_{w}\left(\Gamma_{1}\right)$ of $\Gamma_{1}$ is $d_{w}-a$. In particular $a=0$ if and only if $\varepsilon$ is a loop at $p$, and $a=1$ if and only if $p$ is an endpoint of $\varepsilon$ and $\varepsilon$ is not a loop. The graph $\Gamma_{1}$ has at most two lollipops at the basepoint, say there are $b$ of them, so $b \in\{0,1,2\}$. Let $\Gamma_{2}$ be the graph obtained by removing all lollipops at $p$ in $\Gamma_{1}$. Then the weighted degree $d_{w}\left(\Gamma_{2}\right)$ of $\Gamma_{2}$ is the same as $\Gamma_{1}$, the undistinguished rank is $n-1$, and there are $m-b$ distinguished cycles. By induction, $m-b \leq 2\left(d_{w}-a\right)$, so $m \leq 2 d_{w}-(2 a-b)$. It now suffices to show that $2 a \geq b$. If $a=0$ then $b=0$, so suppose $a>0$. Then the only case to check is when $b=2$. But then $p$ cannot be an endpoint of $\varepsilon$, so $a=2$ and the result follows. We remark that the stronger statement $a \geq b$ even holds.

Lastly suppose that $\Gamma$ has no lollipops or double lollipops at $p$. Let $b \in\{0,1,2\}$ be the number of lollipops in $\Gamma_{1}$ and $c \in\{0,1,2\}$ the number of double lollipops in $\Gamma_{1}$, so $b+c \in\{0,1,2\}$. Let $\Gamma_{3}$ be the graph obtained by removing all lollipops and double lollipops at $p$ in $\Gamma_{1}$. Let $a \in\{0,1,2,3,4\}$ be such that $\Gamma_{3}$ has weighted 
degree $d_{w}-a$. Again, $a=0$ if and only if $\varepsilon$ is a loop at $p$. Also, if $\varepsilon$ is not a loop but $p$ is an endpoint of $\varepsilon$ then $a=1+c$, and otherwise $a=2+c$. See Figure 3 for some examples. By the induction hypothesis $m-(b+2 c) \leq 3\left(d_{w}-a\right) / 2$, so $m \leq 3 d_{w} / 2-(3 a / 2-(b+2 c))$. It now suffices to show that $3 a \geq 2 b+4 c$. If $a=0$ then $b=c=0$, so suppose $a>0$. If $p$ is an endpoint of $\varepsilon$ then $b+c \leq 1$ and $a=1+c$, so $2 b+4 c \leq 2+2 c=2 a<3 a$. Now suppose $p$ is not an endpoint of $\varepsilon$, so $b+c \leq 2$ and $c=a-2$. Then $2 b+4 c \leq 4+2 c=2 a<3 a$ and we are done. Again, we find that a stronger statement holds, namely $a \geq b+2 c$.

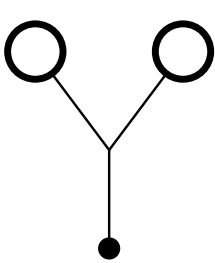

Type 1

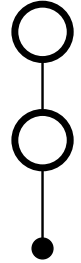

Type 2

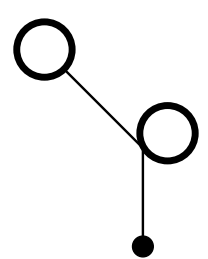

Type 3

Figure 3

Remark 4.4 In the last two paragraphs of the proof, it is interesting that the induction would have run even with sharper bounds. In fact, whatever the best possible bound is for the $n=0$ case automatically extends to all cases, as long as the slope is not less than 1. In particular, we can detect wedge summands that are $r$-iterated lollipop wedges, with increasingly better bounds as $r$ grows. Ultimately, we find that whenever $m>d_{w}$, there is always some nontrivial wedge summand that is an $r$-iterated lollipop wedge for some $r$. However, since we currently do not have a way to make use of this fact to get better bounds for homological stability, we will content ourselves with just detecting lollipops and double lollipops.

Proposition 4.5 (Stability in $n$ ) The map

$$
v: \nabla K_{n, k}^{m} / G_{n}^{m} \hookrightarrow \nabla K_{n+1, k}^{m} / G_{n+1}^{m}
$$

is a homeomorphism for $2 k+m<n+1$ and a homotopy equivalence for $\frac{3 k+m}{2}<n+1$.

Proof The proof is very similar to the proof of Proposition 5.4 in [9]. If $2 k+m<n+1$ then every $\Gamma$ in $\nabla K_{n+1, k}^{m} / G_{n+1}^{m}$ has a loop at $p$, so $v$ is a homeomorphism. Now suppose $(3 k+m) / 2<n+1$, and let $\Gamma$ be a vertex not in the image of $v$. Then $\Gamma$ has no loops at $p$ but does have at least one theta graph wedge summand. Let $\Theta$ be the subgraph of $\Gamma$ consisting of all such theta graphs at $p$, say there are $r \geq 1$ of 
them. Then $\Gamma=\Theta \vee \Gamma^{\prime}$, for some $\Gamma^{\prime}$ with rank $n+m+1-2 r$. Now, the open star of $\Gamma$ in $\nabla K_{n+1, k}^{m} / G_{n+1}^{m}$ is the product of open stars of $\Theta$ in $\nabla K_{2 r, r}^{0} / G_{2 r}^{0}$ and $\Gamma^{\prime}$ in $\nabla K_{n+1-2 r, k-r}^{m} / G_{n+1-2 r}^{m}$. The former consists of a single simplex, since all nonloop edges in $\Theta$ are equivalent under automorphisms of $\Theta$; moreover, every other vertex of this star has lower weighted degree since blowing down any edge reduces $d_{w}$ by 1 . So, collapsing any nonloop edge of $\Theta$ gives a deformation retraction of the star of $\Gamma$ into the image of $v$.

As a remark, in [10] Hatcher and Vogtmann give some bounds to detect wedge summands of higher degree, and the possibility of collapsing these in a similar way to the theta wedge summands is examined. In the present situation though, this collapse could cause $p$ to become distinguished, which is a problem. Hence we cannot immediately improve the bound to $(5 k+m) / 4<n+1$, as was done for the $m=0$ case in [10]. It seems likely that we could nonetheless improve this bound by directly inspecting examples with low (weighted) degree, in the spirit of [10], but we leave this for future work.

Proposition 4.6 (Stability in $m$ ) Let $\nabla Q_{n, k}^{m}:=\nabla K_{n, k}^{m} / \Sigma$ Aut $_{n}^{m}$. The map

$$
\mu: \nabla Q_{n, k}^{m} \hookrightarrow \nabla Q_{n, k}^{m+1}
$$

is a homeomorphism for $2 k<m+1$ and a homotopy equivalence for $3 k / 2<m+1$.

Proof If $2 k<m+1$ then every $\Gamma$ in $\nabla Q_{n, k}^{m+1}$ has a lollipop at $p$, so $\mu$ is a homeomorphism. Now suppose $3 k / 2<m+1$, and let $\Gamma$ be a vertex not in the image of $\mu$. Then $\Gamma$ has no lollipops at $p$ but does have at least one double lollipop. Let $\Lambda \Lambda$ be the subgraph of $\Gamma$ consisting of all double lollipops at $p$, say there are $r \geq 1$ of them. Then $\Gamma=\Lambda \Lambda \vee \Gamma^{\prime}$, for some $\Gamma^{\prime}$ with rank $n+m+1-2 r$. The open star of $\Gamma$ in $\nabla Q_{n, k}^{m+1}$ is the product of open stars of $\Lambda \Lambda$ in $\nabla Q_{0, r}^{2 r}$ and $\Gamma^{\prime}$ in $\nabla Q_{n-2 r, k-r}^{m+1}$. We claim that there is a retraction of the former that yields a retraction of the star of $\Gamma$ into the image of $\mu$, similar to the previous proof. Consider the height function $h$ from Section 3, thought of on $\nabla K_{0, r}^{2 r}$, and note that since $h$ only depends on $\rho$ inasmuch as $\rho$ determines which cycles are distinguished, $h$ descends to a function $\bar{h}$ on $\nabla Q_{0, r}^{2 r}$. Since $\nabla Q_{0, r}^{2 r}$ is not simplicial we think of $\bar{h}$ as a height function in the sense of Bux [3]. It now suffices to show that the descending link $\overline{\mathrm{k} \downarrow}(\Gamma)$ is contractible.

There are three homeomorphism types of double lollipops, depending on where the first lollipop is wedged to the second. If it is wedged to a point in the interior of the stick, call this Type 1 . If it is wedged to a point on the distinguished cycle not in the stick, call this Type 2 . If it is wedged to the vertex shared by the loop and the 
stick call this Type 3. See Figure 4. If $\Lambda \Lambda$ has a double lollipop of Type 1 then blowing down the edge connecting the wedge point to $p$ is descending (with essential term $\left.d_{0}\right)$. Moreover, every simplex in $\overline{\mathrm{lk} \downarrow}(\Gamma)$ is compatible with this move since descending blow-ups cannot affect double lollipops of Type 1, so it is a cone point of $\overline{\mathrm{kk} \downarrow}(\Gamma)$. Next, if $\Lambda \Lambda$ has a double lollipop of Type 2, then blowing down either edge connecting the wedge point to the top of the stick is descending (with essential term $d_{0}$ ). These edges differ by a homeomorphism of $\Gamma$, so they actually correspond to the same blow-down. Again, every simplex in $\overline{\mathrm{k} \downarrow}(\Gamma)$ is compatible with this move since descending blow-ups cannot affect double lollipops of Type 2, so it is a cone point of $\overline{\mathrm{lk} \downarrow}(\Gamma)$. Finally suppose $\Lambda \Lambda$ has a double lollipop of Type 3 . Consider the blow-up that pushes the base of the first cycle away from the wedge point, creating a double lollipop of Type 1. This is descending, with essential term $m_{1}$, and since descending (admissible) blow-downs cannot affect double lollipops of Type 3, it is a cone point for $\overline{\mathrm{k} \downarrow}(\Gamma)$. We conclude that attaching $\Gamma$ does not change the homotopy type, by [3, Lemma 4], so the result follows.

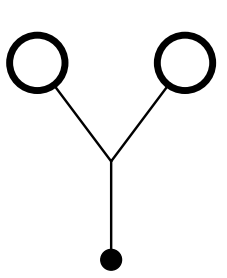

Type 1

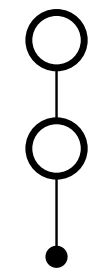

Type 2

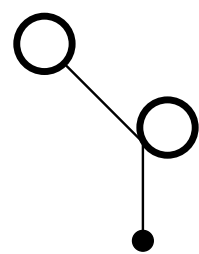

Type 3

Figure 4: Types of double lollipops

There is evidence to suggest that the descending links $\overline{\mathrm{lk} \downarrow}(\Gamma)$ are always contractible whenever there is a nontrivial wedge summand that is an iterated lollipop wedge. As indicated by Remark 4.4, this would imply that $\mu$ is a homotopy equivalence whenever $k \leq m$. From this we would also recover the fact that $\Sigma \mathrm{Aut}_{0}^{m}$ has trivial rational homology, shown independently by Griffin and Wilson [8; 19]. For now though, we will content ourselves with the double lollipop situation.

Before proving our stability theorems, the reader may want a hint as to why we get $n-$ stability for both $\Sigma$ Aut $_{n}^{m}$ and $P \Sigma$ Aut $_{n}^{m}$, but $m$-stability only for $\Sigma$ Aut $_{n}^{m}$. The key is, when looking at $P \sum \mathrm{Aut}_{n}^{m}$, the distinguished cycles in the orbit space are distinguishable from each other; they each have a different "color," so to speak. Thus when we look for features at the basepoint, we would be hunting for, say, a lollipop of one specific color, and this is too much to ask for. In the nonpure case, all the distinguished cycles have the same color so all we care about is finding a lollipop at the basepoint, period. 
Since $v$ is natural with respect to $G_{n}^{m} \hookrightarrow G_{n+1}^{m}$ and $\mu$ is natural with respect to $\Sigma \operatorname{Aut}_{n}^{m} \hookrightarrow \Sigma \operatorname{Aut}_{n}^{m+1}$, we can now prove our main stability results.

Proof of Theorem 1.1 We know that for $0 \leq k<N$, if $(3 k+m) / 2<n+1$ then

$$
H_{i}\left(G_{n}^{m} ; \mathbb{Q}\right) \rightarrow H_{i}\left(G_{n+1}^{m} ; \mathbb{Q}\right)
$$

is an isomorphism for all $i<k$, by Lemma 4.1 and Proposition 4.5. Assume that $n \geq(3(i+1)+m) / 2$, so in particular $n \geq 2$, and set $k=i+1$. Then $(3 k+m) / 2<n+1$ and $k \leq(2 n-m) / 3$, which is less than $N$ since $n \geq 2$. The result now follows.

Note that when $m=0$, so $G_{n}^{0}=\operatorname{Aut}\left(F_{n}\right)$, we recover the stability bound for $\operatorname{Aut}\left(F_{n}\right)$ given in [9], though not the improved one given in [10].

Proof of Theorem 1.2 We know that for $0 \leq k<N$, if $3 k / 2<m+1$ then

$$
H_{i}\left(\Sigma \mathrm{Aut}_{n}^{m} ; \mathbb{Q}\right) \rightarrow H_{i}\left(\Sigma \mathrm{Aut}_{n}^{m+1} ; \mathbb{Q}\right)
$$

is an isomorphism for all $i<k$, by Lemma 4.1 and Proposition 4.6. If $n=0$ then the homology groups are all 0 by [8; 19], so we can assume $n \geq 1$. Suppose $m>(3 i+1) / 2$, so in particular $m \geq 1$, and set $k=i+1$. Then $3 k / 2=3(i+1) / 2<m+1$, and also since $n, m \geq 1$ we get $k<(2 m+2) / 3 \leq 2 n+m-1=N$, so $k<N$. The result now follows.

\section{Connectivity}

The rest of this paper is devoted to proving Proposition 5.14, that $1 \mathrm{k} \downarrow(\Gamma)$ is $\left(d_{w}(\Gamma)-1\right)$ spherical. In reading these subsections, the reader may find it helpful to refer to the corresponding sections in McEwen and Zaremsky [16], which cover what amounts here to the classical $m=0$ case.

We first collect some natural definitions that will be used in these subsections, including the important notion of a decisive edge in a graph.

Definition 5.1 (Edges in graphs) For an edge $\varepsilon$ in a basepointed graph $\Gamma$ with vertices $v$ and $v^{\prime}$, we call $\varepsilon$ horizontal if $d(p, v)=d\left(p, v^{\prime}\right)$. Otherwise we call $\varepsilon$ vertical. If $\varepsilon$ is vertical, by comparing $d(v, p)$ and $d\left(v^{\prime}, p\right)$ we get a natural notion of the top vertex and bottom vertex of $\varepsilon$. A half-edge may also have either a top or a bottom. If a vertex $v$ has only one incident vertical edge $\varepsilon$ with $v$ as its top, we call that edge decisive at $v$. In other words, if every minimal length path from $v$ to $p$ begins with $\varepsilon$, then $\varepsilon$ is decisive at $v$. If an edge $\varepsilon$ in $\Gamma$ is decisive at its top vertex we call it a decisive edge. For example any separating edge is decisive. 


\section{Outline of the section}

There are many technical arguments in this section, so we record here the important steps of the proof. The descending link $\operatorname{lk} \downarrow(\Gamma)$ is the join of the d-down link and d-up link. We analyze the d-down link first, modeled as the geometric realization of the poset of good admissible forests in $\Gamma$, denoted $P(\Gamma)$. The notion of a forest being good is defined in Definition 5.2, along with the important related notion of it being arcfree or arced. The main result of the subsection on the d-down link is the following.

Proposition 5.8 (Homotopy type of the d-down link) $P(\Gamma)$ is homotopy equivalent to a (possibly empty) wedge of spheres of dimension $V-c-2$, where $V$ is the number of vertices in $\Gamma$.

To prove this, we attempt to relate the homotopy of $P(\Gamma)$ to that of certain subposets $P_{0}(\Gamma, \varepsilon) \subseteq P_{1}(\Gamma, \varepsilon)$, where $\varepsilon$ is a cleverly chosen edge (called an optimal edge). For now we denote these by $P_{0}$ and $P_{1}$ for brevity. The easier of these to analyze is $P_{0}$. If $\varepsilon$ is distinguished, $P_{0}$ is contractible (Lemma 5.4). The case when $\varepsilon$ is not distinguished is dealt with during the induction argument in the proof of Proposition 5.8, by realizing that $P_{0}(\Gamma, \varepsilon)$ is isomorphic to $P(\Gamma \backslash \varepsilon)$; the latter is well defined since $\varepsilon$ is undistinguished. The connection between $P_{1}(\Gamma)$ and $P(\Gamma)$ is established in Lemma 5.7. The remaining step, and the hardest, is to establish the connection between $P_{0}$ and $P_{1}$.

Proposition 5.6 (From $P_{0}$ to $P_{1}$ ) Let $\varepsilon$ be an optimal edge. Then $P_{1}(\Gamma, \varepsilon)$ is homotopy equivalent to $P_{0}(\Gamma, \varepsilon)$.

First we enlarge $P_{0}$ to an intermediate poset called $P_{1 / 2}$. If $\varepsilon$ is undistinguished then this actually equals $P_{1}$ and we are done. Now assume $\varepsilon$ is distinguished. We define a new height function $e$ on $P_{1}$ with $P_{1 / 2}$ as a level set, and analyze descending links in $P_{1} \backslash P_{1 / 2}$ with respect to $e$. We claim they are contractible, and prove this by looking at a join factor called the $d$-in link. The key is to retract the d-in link to the star of a cleverly chosen forest (which is a path) $\gamma$.

This finishes the analysis of $P(\Gamma)$, and hence of the d-down link. After showing in Lemma 5.9 that the d-down link of $\Gamma$ is contractible in the special case where $\Gamma$ has a nonbase vertex with an admissible decisive edge, we turn our attention to the d-up link of $\Gamma$. This part does not deviate much from the corresponding part of [16].

Lemma 5.13 (Homotopy type of the d-up link) Suppose $\Gamma$ has no nonbase vertices with an admissible decisive edge. Moreover suppose every base vertex has minimal valency. Then the d-up link of $\Gamma$ is homotopy equivalent to $\bigvee S^{d_{0}-V}$. 
In Lemma 5.10 we decompose the d-up link into a join of complexes BU $\downarrow(v)$ of descending blow-ups at vertices $v$. These can be modeled as complexes $\Sigma \downarrow(v)$ of certain partitions of $\{1, \ldots, n\}$. If $v$ is not a base vertex, $\Sigma \downarrow(v)$ is a wedge of spheres (Lemma 5.11). If $v$ is a base vertex, the complex $\Sigma \downarrow(v)$ is "usually" contractible (Lemma 5.12). The remaining cases are dealt with in the proof of Lemma 5.13.

With the homotopy types of the d-down link and d-up link in hand, we conclude that the descending link $1 \mathrm{k} \downarrow(\Gamma)$, being their join, is highly spherical.

This ends the outline. Now we begin the details.

\subsection{Connectivity of the d-down link}

In this section we analyze the d-down link of $\Gamma$. In order to get an induction to run in the proof of Proposition 5.8, we will need to lift the restriction on the valency of vertices. Our height function $h$ does not work well with such graphs though, for instance the fact that nonbasepoint vertices have valency at least 3 was crucial to the proof in Lemma 3.2 that blowing down a forest $F$ either increases $n_{D(F)}$ or decreases $d_{D(F)}$. Thanks to Lemma 3.6 though, we have a condition on forests that is equivalent to being descending for graphs $\Gamma \in \Delta K_{n}^{m}$, and does not refer to the functions $n_{i}$ or $d_{i}$. For lack of a more clever name, we will call such forests good (defined below). For the rest of this subsection, $\Gamma$ is a connected graph with basepoint $p$ and $m$ disjoint distinguished cycles, with no restriction on the valency of vertices. The definitions of $\Lambda_{i}$ and $m_{i}$ remain valid, and are as given previously. A reduced, non-self-intersecting edge path $\gamma$ in $\Gamma$ will be called an arc if both of its endpoints lie in $\Lambda_{D(\gamma)}$.

Definition 5.2 (Good forests) Let $F$ be an admissible forest in $\Gamma$. Define

$$
\Delta m_{i}(\Gamma, F):=m_{i}(\Gamma / F)-m_{i}(\Gamma)
$$

for any $i$. Now let $i:=D(F)$. If $\Delta m_{i}(\Gamma, F)<0$ call $F$ base-decreasing, if $\Delta m_{i}(\Gamma, F)>0$ call $F$ base-increasing and if $\Delta m_{i}(\Gamma, F)=0$ call $F$ base-preserving. If $F$ connects vertices in $\Lambda_{i}$, or equivalently if $F$ contains an arc $\gamma$ with $D(\gamma)=D(F)$, call $F$ arced. If $F$ does not connect vertices in $\Lambda_{i}$, call $F$ arcfree. Finally, if $F$ is base-decreasing, or if it is base-preserving and arcfree, call $F$ good. A forest is bad if it is not good.

Lemma 3.6 says that for any $\Gamma \in \Delta K_{n}^{m}$, a forest $F$ in $\Gamma$ is descending if and only if it is good.

There are a few important technical observations about some "basic" admissible forests that we collect here. 
Observation 5.3 (Good/bad edges and distinguished paths) Let $\varepsilon$ be an admissible forest that consists of one edge. Let $\gamma$ be an admissible forest that consists of a reduced, non-self-intersecting edge path contained in a distinguished cycle $C$.

(i) Suppose $\varepsilon$ is vertical. Then it is arcfree and cannot be base-decreasing. If it is distinguished, then it is base-preserving and hence good.

(ii) Suppose $\varepsilon$ is horizontal. Then it is arced and cannot be base-increasing. If it is base-decreasing then it must be distinguished. Hence $\varepsilon$ is good if and only if it is distinguished and connects two base vertices.

(iii) The distinguished edge path $\gamma$ is bad if it is arced and $D(\gamma)>i_{C}$, and otherwise is good. In any case $\gamma$ is not base-increasing.

Proof Item (i) follows by definition, and by the observation that a vertical distinguished edge cannot be base-increasing. For item (ii), note that if $\varepsilon$ is base-decreasing then it connects base vertices, and then since it is admissible, it must be distinguished. The other points follow by definition. For item (iii), if $D(\gamma)>i_{C}$ then since $\gamma \subseteq C$, blowing down $\gamma$ does not change $m_{D(\gamma)}$; hence if $\gamma$ is arced then it is bad by definition. If $\gamma$ is arcfree then it is base-preserving, and hence good by definition. For the final case, if $\gamma$ is arced and $D(\gamma)=i_{C}$, then $\gamma$ is base-decreasing and hence good.

See Figure 5 for some examples of item (iii) in the observation.

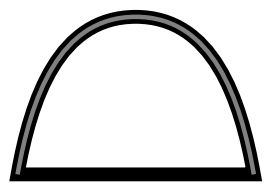

good

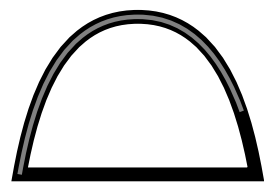

good

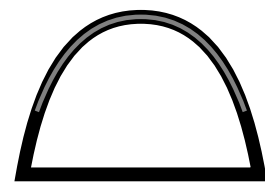

bad

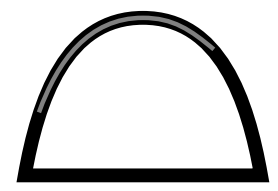

good

Figure 5: Good and bad distinguished edge paths

Posets of forests Let $P(\Gamma)$ be the poset of good admissible forests in $\Gamma$, ordered by inclusion. For $\Gamma \in \Delta K_{n}^{m}$, the d-down link of $\Gamma$ is the geometric realization $|P(\Gamma)|$ of $P(\Gamma)$, so the goal of this section is to calculate the homotopy type of $|P(\Gamma)|$. For the rest of this section we will omit the vertical bars, and just refer to $P(\Gamma)$ as having a homotopy type. For each edge $\varepsilon$ of $\Gamma$, let $P_{1}(\Gamma, \varepsilon)$ be the poset of all good admissible forests except the forest just consisting of $\varepsilon$, and let $P_{0}(\Gamma, \varepsilon) \subseteq P_{1}(\Gamma, \varepsilon)$ be the poset of good admissible forests that do not contain $\varepsilon$. Whenever $\Gamma$ and $\varepsilon$ are understood from context we will just write $P, P_{1}$ and $P_{0}$. We call $P_{1}(\Gamma, \varepsilon)$ the deletion of $\varepsilon$, and $P_{0}(\Gamma, \varepsilon)$ the strong deletion of $\varepsilon$. 
Lemma 5.4 (Strong deletion of distinguished edge) For an admissible distinguished edge $\varepsilon, P_{0}(\Gamma, \varepsilon)$ is contractible.

Proof Let $C$ be the distinguished cycle containing $\varepsilon$, and let $\phi$ be the forest consisting of all edges of $C$ other than $\varepsilon$. Since $D(\phi)=i_{C}, \phi$ is good by Observation 5.3(iii). Let $f: P_{0} \rightarrow P_{0}$ be given by

$$
F \mapsto F \cup \phi .
$$

We claim that for $F \in P_{0}, F \cup \phi$ is an admissible good forest, so $f$ is well defined. Since $\varepsilon \notin F$ and $F$ is admissible, we see that $F \cup \phi$ is an admissible forest (Lemma 2.2). Let $\phi^{\prime}$ be the image of $\phi$ in $\Gamma / F$, so

$$
\Gamma / F \cup \phi=(\Gamma / F) / \phi^{\prime} .
$$

By the same argument as for $\phi, \phi^{\prime}$ is good. Now, $F$, being good, is by definition either base-decreasing, or else base-preserving and arcfree. Since $\phi^{\prime}$ is good, if $F$ is base-decreasing then so is $F \cup \phi$. In this case the claim follows. Now suppose $F$ is base-preserving and arcfree. If $\phi^{\prime}$ is base-decreasing then so is $F \cup \phi$, and we are done. The other option is that $\phi^{\prime}$ is base-preserving and arcfree (like $F$ ). Then $F \cup \phi$ is base-preserving. We need to show it is arcfree, and then the claim will follow. Suppose not, and let $\gamma \subseteq F \cup \phi$ be an arc. Since $F$ is arcfree, $\gamma \nsubseteq F$, and so the image $\gamma^{\prime}$ of $\gamma$ in $\Gamma / F$ is again an edge path. In fact $\gamma^{\prime}$ is an arc. But $\gamma^{\prime} \subseteq \phi^{\prime}$, so this is impossible. This finishes the proof of the claim, that $F \cup \phi \in P_{0}$.

We conclude that $f$ is well defined, and so it follows from work of Quillen [17, Section 1.5] that $P_{0}$ is contractible.

Optimal edges For an admissible edge $\varepsilon$ with endpoints $v_{1}$ and $v_{2}$, call $\varepsilon$ maximally distant if among all admissible edges, $\varepsilon$ maximizes the quantity $d\left(p, v_{1}\right)+d\left(p, v_{2}\right)$. This quantity is even (resp. odd) if $\varepsilon$ is horizontal (resp. vertical). Hence all maximally distant edges have the same orientation, ie, horizontal or vertical. If a maximally distant edge $\varepsilon$ maximizes the quantity $\Delta m_{D(\varepsilon)}(\Gamma, \varepsilon)$ among all maximally distant edges, call $\varepsilon$ optimal.

Observation 5.5 (Good optimal edges) If there exists a good optimal edge, then either every maximally distant edge is vertical and good, or else every maximally distant edge is horizontal and connects base vertices (and so is good).

Proof Let $\varepsilon$ be a good optimal edge and let $\delta$ be another maximally distant edge, so $\delta$ has the same orientation as $\varepsilon$. If $\varepsilon$ is vertical then $\Delta m_{D(\varepsilon)}(\Gamma, \varepsilon) \geq 0$ (Observation 5.3(i)), but $\varepsilon$ is also good, so this quantity must equal 0 . Then since $\varepsilon$ is optimal, it maximizes 
this quantity, whence $\Delta m_{D(\varepsilon)}(\Gamma, \delta)=0$ and $\delta$ is good. The horizontal case follows immediately from Observation 5.3(ii).

Next we want to relate $P_{0}$ and $P_{1}$. This is the most involved step in the analysis of $P$.

Proposition 5.6 (From $P_{0}$ to $P_{1}$ ) Let $\varepsilon$ be an optimal maximally distant edge. Then $P_{1}(\Gamma, \varepsilon)$ is homotopy equivalent to $P_{0}(\Gamma, \varepsilon)$.

Proof We begin by finding an intermediate poset that is more apparently homotopy equivalent to $P_{0}$. Let $P_{1 / 2}=P_{1 / 2}(\Gamma, \varepsilon)$ be the subcomplex of $P_{1}$ spanned by good admissible forests $F$ for which $F \backslash\{\varepsilon\}$ is again a (nonempty) good admissible forest. Call $P_{1 / 2}$ the sufficiently strong deletion of $\varepsilon$. By definition,

$$
P_{0} \subseteq P_{1 / 2} \subseteq P_{1}
$$

Let $f: P_{1 / 2} \rightarrow P_{1 / 2}$ be given by $F \mapsto F \backslash\{\varepsilon\}$. This is a well defined poset map that is the identity on its image $P_{0}$, and so induces a homotopy equivalence between $P_{1 / 2}$ and $P_{0}$ by [17, Section 1.3]. It remains to relate $P_{1 / 2}$ to $P_{1}$.

Case 1: Undistinguished optimal edge First suppose that $\varepsilon$ is undistinguished, and we claim that $P_{1 / 2}=P_{1}$. Let $F \in P_{1}$ and let $i:=D(F)$. We want to show that $F \backslash\{\varepsilon\}$ is good. We may assume $\varepsilon$ is (properly) contained in $F$, which since $\varepsilon$ is maximally distant tells us that $D(F \backslash\{\varepsilon\})=i$. If $\varepsilon^{\prime}$ is the image of $\varepsilon$ in $\Gamma /(F \backslash\{\varepsilon\})$ then $\varepsilon^{\prime}$ is undistinguished, and so cannot be base-decreasing (Observation 5.3(i) and (ii)). Hence

$$
\Delta m_{i}(\Gamma, F)=\Delta m_{i}\left(\Gamma /(F \backslash\{\varepsilon\}), \varepsilon^{\prime}\right)+\Delta m_{i}(\Gamma, F \backslash\{\varepsilon\}) \geq \Delta m_{i}(\Gamma, F \backslash\{\varepsilon\}) .
$$

If $F$ is arcfree then $F \backslash\{\varepsilon\}$ is too. From this fact and the above equation, we conclude (from the definition of good) that if $F$ is good then so is $F \backslash\{\varepsilon\}$. We remark that so far we have not used the hypothesis that $\varepsilon$ is optimal, just that it is maximally distant.

Case 2: Distinguished optimal edge Now assume $\varepsilon$ is distinguished, so we know $\Delta m_{D(\varepsilon)}(F, \varepsilon) \leq 0$ (Observation 5.3(iii)). We have to do a bit more work in this case. Define a height function $e$ on $P_{1}$ as follows. For $F \in P_{1}$, if $F \in P_{1 / 2}$ set $e(F)=0$ and otherwise let $e(F)$ be the number of edges in $F$. Since adjacent vertices (forests) in $P_{1} \backslash P_{1 / 2}$ have different $e$ values, we can build up from $P_{1 / 2}$ to $P_{1}$ by gluing in vertices along their descending links. We claim these descending links are contractible, so by [1, Corollary 2.6] the homotopy type does not change, and the result follows. The descending link of $F \in P_{1} \backslash P_{1 / 2}$ is the join of two subcomplexes, which we will call the $d$-out link and the $d$-in link. The d-out link is spanned by forests in $P_{1 / 2}$ containing $F$, and the d-in link by forests in $P_{1}$ properly contained in $F$. In words, the d-in link is the complex of good admissible forests properly contained in $F$. It suffices to show that the d-in link is contractible. 
Calculating $\Delta m_{i}$ A forest $F$ in $P_{1}$ but not in $P_{1 / 2}$ is characterized by $F$ being good and $F \backslash\{\varepsilon\}$ being bad. This is a relatively specific situation, and we will be able to restrict the possibilities quite a bit. First of all, $\varepsilon \subseteq F$, and $\varepsilon$ is maximally distant so $D(F \backslash\{\varepsilon\})=i:=D(F)$. Now we claim the following.

Claim The forests $F$ and $F \backslash\{\varepsilon\}$ are arced, and

$$
\begin{aligned}
\Delta m_{i}(\Gamma, F) & =-1, \\
\Delta m_{i}\left(\Gamma /(F \backslash\{\varepsilon\}), \varepsilon^{\prime}\right) & =-1, \\
\Delta m_{i}(\Gamma, F \backslash\{\varepsilon\}) & =0 .
\end{aligned}
$$

Proof of the claim Consider again the equation

$$
\Delta m_{i}(\Gamma, F)=\Delta m_{i}\left(\Gamma /(F \backslash\{\varepsilon\}), \varepsilon^{\prime}\right)+\Delta m_{i}(\Gamma, F \backslash\{\varepsilon\}),
$$

where $\varepsilon^{\prime}$ is the image of $\varepsilon$ in $\Gamma /(F \backslash\{\varepsilon\})$. Since $F$ is good and $F \backslash\{\varepsilon\}$ is bad, $\Delta m_{i}(\Gamma, F) \leq 0$ and $\Delta m_{i}(\Gamma, F \backslash\{\varepsilon\}) \geq 0$ by definition. Also, $\Delta m_{i}\left(\Gamma /(F \backslash\{\varepsilon\}), \varepsilon^{\prime}\right)$ can only be $-1,0$ or 1 since $\varepsilon^{\prime}$ is a single edge. In fact it cannot be 1 , since $\varepsilon^{\prime}$ is distinguished (Observation 5.3(i) and (ii)). If it equals 0 then all the terms in the equation are 0 , but then the definitions of good and bad necessitate that $F$ is arcfree and $F \backslash\{\varepsilon\}$ is arced, which is absurd. Thus $\Delta m_{i}\left(\Gamma /(F \backslash\{\varepsilon\}), \varepsilon^{\prime}\right)=-1$. In particular $D\left(\varepsilon^{\prime}\right)=i$ in $\Gamma /(F \backslash\{\varepsilon\})$ (Lemma 3.2).

Now there are two possibilities for $\Delta m_{i}(\Gamma, F)$ and $\Delta m_{i}(\Gamma, F \backslash\{\varepsilon\})$, namely they either equal -1 and 0 (which we want), or else 0 and 1 . We know that $\varepsilon^{\prime}$, being base-decreasing, must connect base vertices (Observation 5.3(i) and (ii)), and so in particular $F$ must be arced, with an arc containing $\varepsilon$ and connecting base vertices. Since $F$ is good it therefore, by definition, must be base-decreasing, and so $\Delta m_{i}(\Gamma, F)=-1$. We now know that the equalities in the claim all hold, and $F$ is arced. That $F \backslash\{\varepsilon\}$ is even arced follows by definition, now that we know it is base-preserving and bad.

Having understood the situation sufficiently, we now hunt for a way to retract the d-in link to a point.

A crucial arc in $\boldsymbol{F}$ Let $C$ be the distinguished cycle containing $\varepsilon$. Since $\varepsilon \subseteq F$ and $F$ is admissible, we know by Lemma 2.2 that $F \cap C$ is a forest (ie, it does not have isolated vertices). Let $\gamma^{\prime}$ be the connected edge path in $F \cap C$ containing $\varepsilon$. By the proof of the claim, $\gamma^{\prime}$ must contain an arc at level $D(F)$ that in turn contains $\varepsilon$. Let $\gamma$ be the shortest arc in $\gamma^{\prime}$ containing $\varepsilon$ with $D(\gamma)=D(F)$. If $\gamma=\varepsilon$ then $D(F)=D(\varepsilon)$, and $\varepsilon$ being both an arc and an optimal edge implies that it, and so every edge of $F$, is horizontal and connects base vertices (Observation 5.5). Hence 
$F \backslash\{\varepsilon\}$ is base-decreasing, which we know is not the case. We can therefore assume $\gamma$ properly contains $\varepsilon$. According to Observation 5.3(iii), $\gamma$ is base-decreasing, hence good, and $\gamma \backslash\{\varepsilon\}$ is nonbase-increasing. By minimality of $\gamma, \gamma \backslash\{\varepsilon\}$ is also arcfree, hence good. Since $F \backslash\{\varepsilon\}$ is bad, this means $\gamma$ does not equal $F$. Hence $\gamma$ is a good, admissible proper subforest of $F$, so $\gamma$ is in the d-in link of $F$. See Figure 6 for an idea of $\gamma^{\prime}$ and $\gamma$.
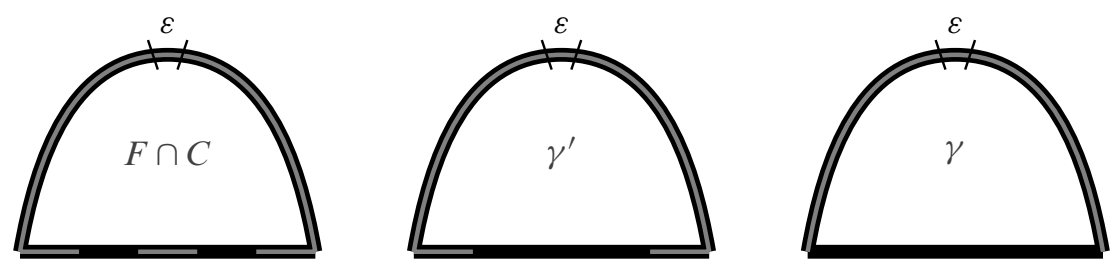

Figure 6: The forest $F \cap C$, and edge paths $\gamma^{\prime}$ and $\gamma$

Contractibility of the d-in link The idea now is to retract the d-in link to the relative star of $\gamma$. We claim that for any $F^{\prime}$ in the d-in link, $F^{\prime} \cup \gamma$ is also in the d-in link. The things to show are that it is admissible, good, and does not equal all of $F$. First note that $F^{\prime} \cup \gamma$ is admissible, since it is contained in $F$. Next we want to see that it is good. If $\gamma \subseteq F^{\prime}$ there is nothing to show, so we can assume rather that the image of $\gamma$ in $\Gamma / F^{\prime}$ is an arc, which necessarily connects base vertices and so is base-decreasing. Since $F^{\prime}$ is good we conclude that $F^{\prime} \cup \gamma$ is base-decreasing, and so is also good.

It remains only to show that $F^{\prime} \cup \gamma \neq F$.

Claim For any $\varnothing \neq \delta \subseteq \gamma, F \backslash \delta$ is bad.

This can phrased colloquially as: if removing $\varepsilon$ from $F$ turns it bad, then removing any part of $\gamma$ from $F$ turns it bad. Since $F^{\prime}$ is good, this will then imply that it is not of the form $F \backslash \delta$, and so $F^{\prime} \cup \gamma \neq F$.

Proof of claim Note that if $\varepsilon \notin \delta$ and $F \backslash \delta$ is good, then the connected component of $(F \backslash \delta) \cap C$ containing $\varepsilon$ does not connect base vertices (by minimality of $\gamma$ ). As seen in the previous paragraphs, this was a necessary requirement for a forest to be in $P_{1} \backslash P_{1 / 2}$, so we conclude that, instead, $F \backslash \delta$ is in $P_{1 / 2}$, whence by definition we have that $F \backslash(\delta \cup\{\varepsilon\})$ is good. All of this is to say that, if $F \backslash \delta$ is good then so is $F \backslash(\delta \cup\{\varepsilon\})$. Taking the contrapositive, if $F \backslash(\delta \cup\{\varepsilon\})$ is bad then so is $F \backslash \delta$, so we can assume without loss of generality that $\varepsilon \subseteq \delta$. 
Now, since $F \backslash\{\varepsilon\}$ is arced and $\gamma$ is minimal we have $D(F \backslash \gamma)=i$, and so also $D(F \backslash \delta)=i$. Since the edges of $\gamma$ are distinguished, we know from Observation 5.3(iii) that

$$
\Delta m_{i}(\Gamma, F \backslash \delta) \geq \Delta m_{i}(\Gamma, F \backslash\{\varepsilon\})=0,
$$

so to show $F \backslash \delta$ is bad, it suffices to assume it is arcfree and prove it is base-increasing. We will use the equation

$$
\Delta m_{i}(\Gamma, F \backslash \delta)+\Delta m_{i}\left(\Gamma /(F \backslash \delta),(\delta \backslash\{\epsilon\})^{\prime}\right)=\Delta m_{i}(\Gamma, F \backslash\{\varepsilon\})=0,
$$

where $(\delta \backslash\{\epsilon\})^{\prime}$ is the image of $\delta \backslash\{\epsilon\}$ in $\Gamma /(F \backslash \delta)$. For $F \backslash\{\varepsilon\}$ to be arced and $F \backslash \delta$ to be arcfree, there must exist an arc in $F \backslash\{\varepsilon\}$ containing an edge of $\delta \backslash\{\varepsilon\}$. In particular, $(\delta \backslash\{\varepsilon\})^{\prime}$ is an arced forest consisting of distinguished edges, with an arc connecting base vertices. This must be base-decreasing, again by Observation 5.3(iii), so $\Delta m_{i}\left(\Gamma /(F \backslash \delta),(\delta \backslash\{\epsilon\})^{\prime}\right)<0$ and the above equation becomes

$$
\Delta m_{i}(\Gamma, F \backslash \delta)>0,
$$

so $F \backslash \delta$ is bad and we are done.

This finishes the proof of the claim, so now we know $F^{\prime} \cup \gamma$ is in the d-in link of $F$. In particular the $\mathrm{d}$-in link is contractible by [17, Section 1.5]. Then this finishes the proof that $P_{1}(\Gamma, \varepsilon) \simeq P_{0}(\Gamma, \varepsilon)$.

Next we want to relate $P_{1}$ to $P$.

Decomposing $\boldsymbol{P}$ using $\boldsymbol{\varepsilon}$ In general if $\varepsilon$ is any admissible good edge, then we have

$$
\begin{aligned}
P(\Gamma)= & P_{1}(\Gamma, \varepsilon) \cup \operatorname{st}(\varepsilon), \\
& P_{1}(\Gamma, \varepsilon) \cap \operatorname{st}(\varepsilon)=\operatorname{lk}(\varepsilon),
\end{aligned}
$$

where star and link here are taken in $P(\Gamma)$. The results up to this point provide tools to analyze $P_{1}(\Gamma, \varepsilon)$, and the next lemma tells us something about $\operatorname{lk}(\varepsilon)$.

Lemma 5.7 (Links in the d-down link) Let $\varepsilon$ be an optimal edge in $\Gamma$ such that $\varepsilon \in P(\Gamma)$, ie, $\varepsilon$ is good. Let $F$ be an admissible forest properly containing $\varepsilon$. Then $F \in P(\Gamma)$ if and only if $F / \varepsilon \in P(\Gamma / \varepsilon)$. Moreover, $\operatorname{lk}(\varepsilon) \cong P(\Gamma / \varepsilon)$.

Proof Let $i:=D(F)=D(F / \varepsilon)$. Since $\varepsilon$ is good, $\Delta m_{i}(\Gamma, \varepsilon) \in\{-1,0\}$. First suppose that $\Delta m_{i}(\Gamma, \varepsilon)=0$, for example if $D(\varepsilon)>i$. Since $\varepsilon$ is optimal, $F$ is arced if and only if $F / \varepsilon$ is arced. Also,

$$
\Delta m_{i}(\Gamma, F)=\Delta m_{i}(\Gamma / \varepsilon, F / \varepsilon)+\Delta m_{i}(\Gamma, \varepsilon),
$$


so $\Delta m_{i}(\Gamma, F)=\Delta m_{i}(\Gamma / \varepsilon, F / \varepsilon)$. Hence, $F$ is base-decreasing if and only if $F / \varepsilon$ is, and $F$ is base-preserving and arcfree if and only if $F / \varepsilon$ is, which implies that $F \in P(\Gamma)$ if and only if $F / \varepsilon \in P(\Gamma / \varepsilon)$.

Next suppose $\Delta m_{i}(\Gamma, \varepsilon)=-1$, so $D(\varepsilon)=i$. We claim that in fact $F$ and $F / \varepsilon$ must both be base-decreasing, and hence good. First observe that every maximally distant edge is optimal, since the maximized quantity $\Delta m_{i}(\Gamma, \varepsilon)=-1$ is also minimized. We know that $\varepsilon$, and indeed every maximally distant edge, is horizontal and connects base vertices (Observations 5.3(i), (ii) and 5.5). In particular since $D(\varepsilon)=i$, every edge of $F$ must be maximally distant, and so connects base vertices. Since $F$ has more than one edge, $\Delta m_{i}(\Gamma, F) \leq-2$, so $F$ is base-decreasing. Also,

$$
\Delta m_{i}(\Gamma / \varepsilon, F / \varepsilon)=\Delta m_{i}(\Gamma, F)-\Delta m_{i}(\Gamma, \varepsilon) \leq-2+1=-1,
$$

so $F / \varepsilon$ is base-decreasing.

Now consider the map

$$
f: \operatorname{lk}(\varepsilon) \rightarrow P(\Gamma / \varepsilon)
$$

sending $F$ to $F / \varepsilon$. This is well-defined by the previous paragraphs. We claim that $f$ is bijective. Let $\Phi \in P(\Gamma / \varepsilon)$. There are two forests in $\Gamma$ that map to $\Phi$ under blowing down $\varepsilon$, one that contains $\varepsilon$ and one that does not (already this shows that $f$ is injective). Let $\Phi^{\prime}$ be the one that does, so $\Phi^{\prime} \in \operatorname{lk}(\varepsilon)$ and $f\left(\Phi^{\prime}\right)=\Phi$. If $\Phi$ was admissible then $\Phi^{\prime}$ is too. Also, if $\Phi$ was good then so is $\Phi^{\prime}$, again by the previous paragraphs. So $f$ is an isomorphism.

Let $V$ be the number of vertices in $\Gamma$ and $E_{a d}$ the number of admissible edges. The next two results are generalizations of Proposition 3.2 and Lemma 3.3 from [16]. Recall that $c$ is the number of distinguished cycles not at $p$.

Proposition 5.8 (Homotopy type of the d-down link) $P(\Gamma)$ is homotopy equivalent to a (possibly empty) wedge of spheres of dimension $V-c-2$.

Proof The proof is similar to the proof of Proposition 2.2 in Vogtmann [18] and of Proposition 3.2 in [16]. We induct on the number of admissible edges $E_{a d}$. Since undistinguished loops do not affect $P(\Gamma), V$ or $c$, we may assume there are none. The base case is $E_{a d}=0$, for which $P(\Gamma)$ is empty, ie $S^{-1}$. When $m>0$, if there are no admissible edges then $V=m$ and $c=m-1$. If $m=0$ and there are no admissible edges then $V=1$ and $c=0$. In both cases, $-1=V-c-2$, which finishes the base case.

Now assume $E_{a d}>0$, so in particular there exists a maximally distant edge. Let $\varepsilon$ be an optimal (maximally distant) edge. First suppose that $\varepsilon$ is distinguished. By Lemma 5.4 
and Proposition 5.6, $P_{1}(\Gamma, \varepsilon)$ is contractible. If $\varepsilon$ is bad then $P(\Gamma)=P_{1}(\Gamma, \varepsilon)$ and we are done, so assume $\varepsilon$ is good. Then $\operatorname{lk}(\varepsilon) \cong P(\Gamma / \varepsilon)$ by Lemma 5.7, and $P(\Gamma / \varepsilon)$ has fewer than $E_{a d}$ admissible edges, so by induction $\operatorname{lk}(\varepsilon)$ is $(V-c-3)$-spherical. Since

$$
\begin{aligned}
P(\Gamma)= & P_{1}(\Gamma, \varepsilon) \cup \operatorname{st}(\varepsilon), \\
& P_{1}(\Gamma, \varepsilon) \cap \operatorname{st}(\varepsilon)=\operatorname{lk}(\varepsilon),
\end{aligned}
$$

we conclude that $P(\Gamma)$ is $(V-c-2)$-spherical.

Next suppose that $\varepsilon$ is not distinguished, and is not a separating edge. By the same argument as above, if $\varepsilon$ is good then $\operatorname{lk}(\varepsilon)$ is $(V-c-3)$-spherical (and if $\varepsilon$ is bad then $P=P_{1}$ anyway), so we just need to inspect $P_{1}$, which by Proposition 5.6 is homotopy equivalent to $P_{0}$. Since $\varepsilon$ is not a separating edge, we can remove it from $\Gamma$ and we still have a connected graph with $m$ distinguished cycles and $V$ vertices, and strictly fewer admissible edges. By induction then, $P(\Gamma \backslash \varepsilon)$ is $(V-c-2)$-spherical (since $c$ did not change either). Consider the map

$$
g: P(\Gamma \backslash \varepsilon) \rightarrow P_{0}(\Gamma, \varepsilon)
$$

induced by $\Gamma \backslash \varepsilon \hookrightarrow \Gamma$. Adding $\varepsilon$ to the graph cannot affect whether a forest $F$ in $\Gamma \backslash \varepsilon$ is admissible or not. Also, since $\varepsilon$ is maximally distant, $\varepsilon$ cannot be decisive, so adding $\varepsilon$ to the graph does not change the levels $\Lambda_{i}$. In particular adding $\varepsilon$ cannot affect whether a forest $F$ in $\Gamma \backslash \varepsilon$ is good or bad, so $g$ is an isomorphism. We conclude that $P_{0}(\Gamma, \varepsilon)$ is $(V-c-2)$-spherical, and hence so is $P(\Gamma)$.

Lastly suppose $\varepsilon$ is not distinguished, but is an (admissible) separating edge. If $\varepsilon$ is good then for any $F \in P(\Gamma), F \cup \varepsilon$ is again an admissible good forest. In this case $P(\Gamma)$ is contractible by [17, Section 1.5]. Incidentally, this completely finishes the $m=0$ case. If $\varepsilon$ is bad then it is base-increasing (Observation 5.3(i)) and so its top must be a base vertex. Since $\varepsilon$ is maximally distant, and $\Gamma$ has no undistinguished loops, $\varepsilon$ is the stick of a lollipop $\ell$. The graph $\Gamma \backslash \ell$ has $V-1$ vertices and $c-1$ distinguished cycles not at $p$, and has fewer admissible edges than $\Gamma$. Also, $P_{0}(\Gamma, \varepsilon) \cong P(\Gamma \backslash \ell)$, so by induction, $P(\Gamma)=P_{1}(\Gamma, \varepsilon) \simeq P_{0}(\Gamma, \varepsilon)$ is $(V-1)-(c-1)-2=(V-c-2)-$ spherical.

Lemma 5.9 (Decisive edges) If $\Gamma$ has a nonbase vertex with an admissible decisive edge then $P(\Gamma)$ is contractible.

Proof The proof has essentially the same structure as the previous one. Induct on $E_{a d}$. In the base case, there are no admissible edges, much less admissible decisive edges, so the claim is vacuously true. Now assume $E_{a d}>0$. Let $\varepsilon$ be an optimal maximally 
distant edge, so $P_{1}(\Gamma, \varepsilon)$ and $P_{0}(\Gamma, \varepsilon)$ are homotopy equivalent. We want to find a decisive edge $\eta \neq \varepsilon$ with top a nonbase vertex. Being maximally distant, the only way $\varepsilon$ could be decisive is if it is separating. If $\varepsilon$ is a good separating edge, then $P(\Gamma)$ is already contractible with cone point $\varepsilon$. If $\varepsilon$ is a bad separating edge, then its top is a base vertex. Hence our hypothesis allows us to assume there is a decisive edge $\eta \neq \varepsilon$ with top a nonbase vertex.

Now we want to prove that $P(\Gamma)$ is contractible. First suppose that $\varepsilon$ is distinguished. Then $P_{1}(\Gamma, \varepsilon)$ is contractible, so if $\varepsilon$ is bad we are done. If $\varepsilon$ is good, we still have that $\operatorname{lk}(\varepsilon) \cong P(\Gamma / \varepsilon)$ as in the previous proof. By Observation 5.3(i) and (ii), $\varepsilon$ is either vertical, or is horizontal and connects base vertices. In either case, $\eta$ maps to a decisive edge in $\Gamma / \varepsilon$, with a nonbase vertex for a top, and so $\operatorname{lk}(\varepsilon)$ is contractible by induction. Therefore $P(\Gamma)$ is contractible. Now suppose $\varepsilon$ is not distinguished. Again, $\operatorname{lk}(\varepsilon)$ is contractible if $\varepsilon$ is good, so we just need to inspect $P_{0}(\Gamma, \varepsilon)$. If $\varepsilon$ is not a separating edge we may remove it as in the previous proof and get that $P_{0}(\Gamma, \varepsilon) \cong P(\Gamma \backslash \varepsilon)$ is contractible by induction. The only case remaining is when $\varepsilon$ is a separating edge whose top is a distinguished vertex, so it is the stick of a lollipop $\ell$. Then $\eta$ is still a decisive edge in $\Gamma \backslash \ell$, so $P(\Gamma)=P_{0}(\Gamma, \varepsilon) \cong P(\Gamma \backslash \ell)$ is contractible by induction.

\subsection{Connectivity of the d-up link}

Now consider the d-up link of $\Gamma$. We return to only considering graphs coming from $\Delta K_{n}^{m}$, so all vertices $v \neq p$ are at least trivalent and $p$ is at least bivalent. Let $\mathrm{BU}(v)$ be the poset of all blow-ups at the vertex $v$, and let $\mathrm{BU} \downarrow(v)$ be the poset of descending blow-ups at $v$. We will use the combinatorial framework for graph blow-ups described Culler and Vogtmann in [6] and Vogtmann in [18], so we think of $\mathrm{BU}(v)$ as the poset of compatible partitions of the set of incident half-edges. Let $[n]:=\{1, \ldots, n\}$, and consider partitions of $[n]$ into two blocks. Denote such a partition by $\alpha=\{a, \bar{a}\}$, where $1 \in a$. Distinct partitions $\{a, \bar{a}\}$ and $\{b, \bar{b}\}$ are called compatible if either $a \subset b$ or $b \subset a$. Let $\Sigma(v)$ be the simplicial complex of partitions $\alpha=\{a, \bar{a}\}$ of $[\operatorname{val}(v)]$ into blocks $a$ and $\bar{a}$ such that $a$ and $\bar{a}$ each have at least two elements. (If $v$ is the basepoint $p$, then one block may have size one, since $p$ is allowed to be bivalent.) That is, the vertices of $\Sigma(v)$ are partitions, and a $j$-simplex is given by a collection of $j+1$ distinct, pairwise compatible partitions. Also let $\Sigma \downarrow(v)$ be the subcomplex of $\Sigma(v)$ spanned by descending partitions, ie, partitions that correspond to descending single-edge blow-ups.

For $v \neq p$, the geometric realization $|\mathrm{BU}(v)|$ of $\mathrm{BU}(v)$ is isomorphic to the barycentric subdivision of $\Sigma(v)$. The idea is that a partition describes an ideal edge, ie, an edge blow-up at a vertex, and the blocks $a$ and $\bar{a}$ indicate which half-edges attach to 
which endpoints of the new edge. See [6] and [18] for more details. The geometric realization $|\mathrm{BU} \downarrow(v)|$ contains the barycentric subdivision of $\Sigma \downarrow(v)$ as a subcomplex, and any simplex in $|\mathrm{BU} \downarrow(v)|$ has at least one vertex in $\Sigma \downarrow(v)$. Hence there is a map $|\mathrm{BU} \downarrow(v)| \rightarrow|\mathrm{BU} \downarrow(v)|$ sending each simplex to its face spanned by vertices in $\Sigma \downarrow(v)$, which induces a deformation retraction from $|\mathrm{BU} \downarrow(v)|$ to $\Sigma \downarrow(v)$.

The next lemma relates the d-up link of $\Gamma$ to these complexes $\Sigma \downarrow(v)$. The proof is very similar to the proof of [16, Proposition 4.5].

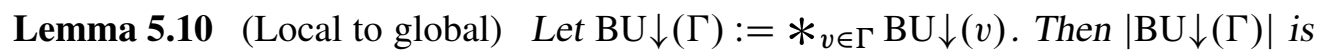
homotopy equivalent to the $d$-up link of $\Gamma$.

Proof For a poset $P$, let $\underline{P}$ be $P \sqcup\{\perp\}$, where $\perp$ is a formal minimal element. Then we have that $P * Q \simeq \underline{P} \times \underline{Q}-\{(\perp, \perp)\}$. Let

$$
U:=\left\{f \in \prod_{v} \underline{\mathrm{BU}}(v)-\left\{(\perp)_{v}\right\} \mid f \text { is descending }\right\},
$$

so the geometric realization $|U|$ is the d-up link. Define a poset map $r: U \rightarrow U$ via

$$
\left(f_{v}\right)_{v} \mapsto\left(\begin{array}{ll}
f_{v} & \text { for } f_{v} \in \mathrm{BU} \downarrow(v), \\
\perp & \text { for } \left.f_{v} \notin \mathrm{BU} \downarrow(v),\right)_{v}
\end{array}\right.
$$

where $f_{v}$ is a blow-up at $v$ in the tuple $f$. This map is well defined since if $f$ is descending then $f_{v}$ must be descending for some $v$. By construction, $r$ is the identity when restricted to $\mathrm{BU} \downarrow(\Gamma)$. Also, $r(f) \leq f$ for all $f \in U$, and so by $[17,1.3]$ this induces a homotopy equivalence between $|U|$ and $|\mathrm{BU} \downarrow(\Gamma)|$.

In particular the d-up link is homotopy equivalent to $*_{v \in \Gamma} \Sigma \downarrow(v)$, so we can analyze the d-up link by looking at the complexes $\Sigma \downarrow(v)$. In light of Lemma 5.9, one important situation is when $v$ is a nonbase vertex with no decisive edges.

Lemma 5.11 (No decisive edges, locally) Suppose $v \neq p$ is a nonbase vertex with no decisive edge. Then $\Sigma \downarrow(v) \simeq \bigvee S^{\operatorname{val}(v)-4}$.

Proof We know that among the half-edges at $v$, at least two correspond to vertical edges with top $v$. Since $v$ is a nonbase vertex, a blow-up at $v$ is descending if and only if it separates some of these half-edges with top $v$. (This is due to Observation 3.3. The essential term will be $n_{d(p, v)}$.) Thus $\Sigma \downarrow(v)$ is isomorphic to the complex denoted $\operatorname{SBU}(v)$ in [16], and the result is immediate from [16, Lemma 4.1, Proposition 4.3]. 
Next we describe one very important case for which the d-up link, and hence $1 \mathrm{k} \downarrow(\Gamma)$ is contractible. If a vertex $v \neq p$ has valency 3 , or if $v=p$ and $\operatorname{val}(v)=2$, we say $v$ has minimal valency. Otherwise we naturally say it has nonminimal valency.

Lemma 5.12 (Contractible case) If $\Gamma$ has a base vertex with nonminimal valency, then the $d$-up link is contractible, and so $1 \mathrm{k} \downarrow(\Gamma)$ is contractible.

Proof Let $v$ be a base vertex with nonminimal valency. By Lemma 5.10 it suffices to show that $\Sigma \downarrow(v)$ is contractible. Label the distinguished half-edges at $v$ by $c_{1}$ and $c_{2}$, and label the undistinguished half-edges by $b_{1}, \ldots, b_{q}$. By hypothesis, $q>1$, unless $v=p$ in which case $q>0$. Let $\alpha_{0}$ be the ideal edge at $v$ that separates $c_{1}, c_{2}$ from all the other half-edges. See Figure 7 for an example. This is a descending blow-up, with essential term $m_{d(p, v)}$. Also, any partition of $\left\{c_{1}, c_{2}, b_{1}, \ldots, b_{q}\right\}$ that separates $c_{1}$ and $c_{2}$ is ascending, so indeed $\Sigma \downarrow(v)$ is contractible with cone point $\alpha_{0}$.
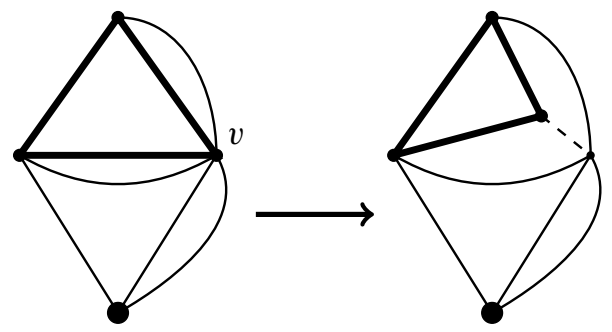

Figure 7: The blow-up at $v$ given by $\alpha_{0}$; here $m_{1}$ goes from 2 to 1

We may now assume every base vertex has minimal valency, and so $\Sigma \downarrow(v)$ is empty for all base vertices $v$. Let $V$ be the number of vertices of $\Gamma$, and recall that here $d_{0}=d_{0}(\Gamma)$ is the degree of $\Gamma$, ie, $d_{0}=2 n+2 m-\operatorname{val}(p)$.

Lemma 5.13 (Homotopy type of the d-up link) Suppose $\Gamma$ has no nonbase vertices with an admissible decisive edge. Moreover suppose every base vertex has minimal valency. Then the d-up link of $\Gamma$ is homotopy equivalent to $\bigvee S^{d_{0}-V}$.

Proof By Lemma 5.10, the d-up link is homotopy equivalent to $*_{v \in \Gamma} \Sigma \downarrow(v)$. Since $\Sigma \downarrow(p)=\varnothing$, this is the same as $*_{v \neq p} \Sigma \downarrow(v)$. Also, we are assuming that each base vertex $u \neq p$ has valency 3 , so $\Sigma \downarrow(u)=\varnothing=S^{\mathrm{val}(u)-4}$. Therefore by Lemma 5.11 the d-up link is homotopy equivalent to

$$
\underset{v \neq p}{*}\left(\bigvee S^{\operatorname{val}(v)-4}\right)
$$


which is a wedge of spheres of dimension $(V-2)+\sum_{v \neq p}(\operatorname{val}(v)-4)$. Observe that

$$
\sum_{v \neq p}(\operatorname{val}(v)-2)=d_{0}
$$

so this dimension equals $(V-2)+d_{0}-2(V-1)=d_{0}-V$.

We can now prove our main result of this section. Here $d_{w}$ is the weighted degree, which recall equals $d_{0}-c$.

Proposition 5.14 (Connectivity of descending links) The descending link $1 \mathrm{k} \downarrow(\Gamma)$ is either contractible or a wedge of spheres of dimension $d_{w}-1$.

Proof Assume that neither the d-up link nor d-down link is contractible. Then every base vertex has minimal valency (Lemma 5.12), and no nonbase vertex of $\Gamma$ has a decisive edge (Lemma 5.9). By Proposition 5.8, $P(\Gamma) \simeq \bigvee S^{V-c-2}$, and by Lemma 5.13 the d-up link is homotopy equivalent to $\bigvee S^{d_{0}-V}$. Hence $1 \mathrm{k} \downarrow(\Gamma)$ is homotopy equivalent to

$$
\left(\bigvee S^{V-c-2}\right) *\left(\bigvee S^{d_{0}-V}\right) \simeq \bigvee S^{V-c-2+d_{0}-V+1}=\bigvee S^{d_{0}-c-1}=\bigvee S^{d_{w}-1}
$$

This completes the proof.

Remark 5.15 (Concluding remarks) We conclude with some questions that now naturally arise. First, the stable rational homology of $\Sigma$ Aut $_{n}^{0}$ in $n$ is trivial, and the rational homology of $\Sigma \mathrm{Aut}_{0}^{m}$ is trivial in every dimension, so it seems likely that the stable homology in $m$ and $n$ is always trivial; is this indeed the case? Some additional evidence for this is Theorem 7.4 in Jensen and Wahl [14], which implies that $H_{1}\left(P \Sigma \operatorname{Aut}_{n}^{m} ; \mathbb{Q}\right)=0$ for any $n>2$ and any $m \geq 0$. Second, there exist examples where $H_{i}\left(\Sigma \mathrm{Aut}_{n}^{0} ; \mathbb{Q}\right)=\mathbb{Q}$, but when can nontrivial rational homology occur in general, eg, if $m>0$ ? This is an interesting question for outer automorphisms as well. Third, when $n=0$ or $m=0$, we have stable integral homology, so an obvious question is whether this holds in general. Fourth, we know that $H_{i}\left(P \Sigma \mathrm{Aut}_{0}^{m} ; \mathbb{Q}\right)$ is not stable in $m$, but as stated above, $H_{1}\left(P \Sigma\right.$ Aut $\left._{n}^{m} ; \mathbb{Q}\right)$ is stably constant 0 for $n>2$. What can we expect in general for $H_{i}\left(P \Sigma \mathrm{Aut}_{n}^{m} ; \mathbb{Q}\right)$ in terms of stability in $m$ ?

\section{References}

[1] M Bestvina, N Brady, Morse theory and finiteness properties of groups, Invent. Math. 129 (1997) 445-470 MR1465330 
[2] KS Brown, Cohomology of groups, Graduate Texts in Mathematics 87, Springer, New York (1982) MR672956

[3] K-U Bux, Orbit spaces of subgroup complexes, Morse theory, and a new proof of a conjecture of Webb, from: "Proceedings of the 1999 Topology and Dynamics Conference", Topology Proc. 24 (1999) 39-51 MR1802676

[4] K-U Bux, R Charney, K Vogtmann, Automorphisms of two-dimensional RAAGS and partially symmetric automorphisms of free groups, Groups Geom. Dyn. 3 (2009) 541-554 MR2529947

[5] D J Collins, Cohomological dimension and symmetric automorphisms of a free group, Comment. Math. Helv. 64 (1989) 44-61 MR982561

[6] M Culler, K Vogtmann, Moduli of graphs and automorphisms of free groups, Invent. Math. 84 (1986) 91-119 MR830040

[7] S Galatius, Stable homology of automorphism groups of free groups, Ann. of Math. 173 (2011) 705-768 MR2784914

[8] J T Griffin, Diagonal complexes and the integral homology of the automorphism group of a free product, Proc. Lond. Math. Soc. 106 (2013) 1087-1120 MR3066749

[9] A Hatcher, K Vogtmann, Cerf theory for graphs, J. London Math. Soc. 58 (1998) 633-655 MR1678155

[10] A Hatcher, K Vogtmann, Rational homology of $\operatorname{Aut}\left(F_{n}\right)$, Math. Res. Lett. 5 (1998) 759-780 MR1671188

[11] A Hatcher, N Wahl, Stabilization for the automorphisms of free groups with boundaries, Geom. Topol. 9 (2005) 1295-1336 MR2174267

[12] A Hatcher, N Wahl, Stabilization for mapping class groups of 3-manifolds, Duke Math. J. 155 (2010) 205-269 MR2736166

[13] C A Jensen, J McCammond, J Meier, The integral cohomology of the group of loops, Geom. Topol. 10 (2006) 759-784 MR2240905

[14] C A Jensen, N Wahl, Automorphisms of free groups with boundaries, Algebr. Geom. Topol. 4 (2004) 543-569 MR2077676

[15] R A McEwen, Homological stability for the groups Out $P(n, t+1), \mathrm{PhD}$ thesis, University of Virginia (2010)

[16] R McEwen, M C B Zaremsky, A combinatorial proof of the Degree theorem in Auter space, to appear in New York J. of Math.

[17] D Quillen, Homotopy properties of the poset of nontrivial p-subgroups of a group, Adv. in Math. 28 (1978) 101-128 MR493916

[18] K Vogtmann, Local structure of some Out $\left(F_{n}\right)$-complexes, Proc. Edinburgh Math. Soc. 33 (1990) 367-379 MR1077791 
[19] J C H Wilson, Representation stability for the cohomology of the pure string motion groups, Algebr. Geom. Topol. 12 (2012) 909-931 MR2928898

Department of Mathematical Sciences, Binghamton University

Binghamton, NY 13902, USA

zaremsky@math. binghamton. edu

http://www . math. binghamton.edu/zaremsky/

Received: 17 January $2013 \quad$ Revised: 21 October 2013 
